\title{
A Study of Quasar Selection in the Supernova Fields of the Dark Energy Survey
}

S. S. Tie ${ }^{1}$, P. Martini ${ }^{1,2}$, D. Mudd ${ }^{1}$, F. Ostrovski ${ }^{3,4}$, S. L. Reed $^{3,4}$, C. Lidman ${ }^{5}$, C. Kochanek ${ }^{1,2}$, T. M. Davis ${ }^{6}$, R. Sharp ${ }^{7}$, S. Uddin $^{8}$, A. King ${ }^{6}$, W. Wester ${ }^{9}$, B. E. Tucker ${ }^{7}$, D. L. Tucker ${ }^{9}$, E. Buckley-Geer ${ }^{9}$, D. Carollo ${ }^{10,11}$, M. Childress ${ }^{7,12}$, K. Glazebrook ${ }^{8}$,

S. R. Hinton ${ }^{6}$, G. Lewis ${ }^{13}$, E. Macaulay ${ }^{6}$, C. R. O’Neill ${ }^{6}$, T. M. C. Abbott ${ }^{14}$, F. B. Abdalla ${ }^{15,16}$, J. Annis ${ }^{9}$, A. Benoit-Lévy ${ }^{15,17,18}$, E. Bertin ${ }^{17,18}$, D. Brooks ${ }^{15}$, A. Carnero Rosel1 ${ }^{19,20}$, M. Carrasco Kind ${ }^{21,22}$, J. Carretero ${ }^{23,24}$, C. E. Cunha ${ }^{25}$, L. N. da Costa ${ }^{19,20}$, D. L. DePoy ${ }^{26}$, S. Desai ${ }^{27}$, P. Doel ${ }^{15}$, T. F. Eifler ${ }^{28}$, A. E. Evrard ${ }^{29,30}$, D. A. Finley ${ }^{9}$, B. Flaugher ${ }^{9}$, P. Fosalba ${ }^{23}$, J. Frieman $^{9,31}$, J. García-Bellido ${ }^{32}$, E. Gaztanaga ${ }^{23}$, D. W. Gerdes ${ }^{30}$, D. A. Goldstein ${ }^{33,34}$, D. Gruen ${ }^{25,35}$, R. A. Gruendl ${ }^{21,22}$, G. Gutierrez ${ }^{9}$, K. Honscheid ${ }^{2,36}$, D. J. James ${ }^{14,37}$, K. Kuehn ${ }^{5}$, N. Kuropatkin ${ }^{9}$, M. Lima ${ }^{19,38}$, M. A. G. Maia ${ }^{19,20}$, J. L. Marshall ${ }^{26}$, F. Menanteau ${ }^{21,22}$, C. J. Miller ${ }^{29,30}$, R. Miquel ${ }^{24,39}$, R. C. Nichol ${ }^{40}$, B. Nord ${ }^{9}$, R. Ogando ${ }^{19,20}$, A. A. Plazas ${ }^{28}$, A. K. Romer ${ }^{41}$, E. Sanchez ${ }^{42}$, B. Santiago ${ }^{19,43}$, V. Scarpine ${ }^{9}$, M. Schubnell ${ }^{30}$, I. Sevilla-Noarbe ${ }^{42}$, R. C. Smith ${ }^{14}$, M. Soares-Santos ${ }^{9}$, F. Sobreira ${ }^{19,44}$, E. Suchyta ${ }^{44}$, M. E. C. Swanson ${ }^{22}$, G. Tarle ${ }^{30}$, D. Thomas ${ }^{40}$, and A. R. Walker ${ }^{14}$

(The DES Collaboration)

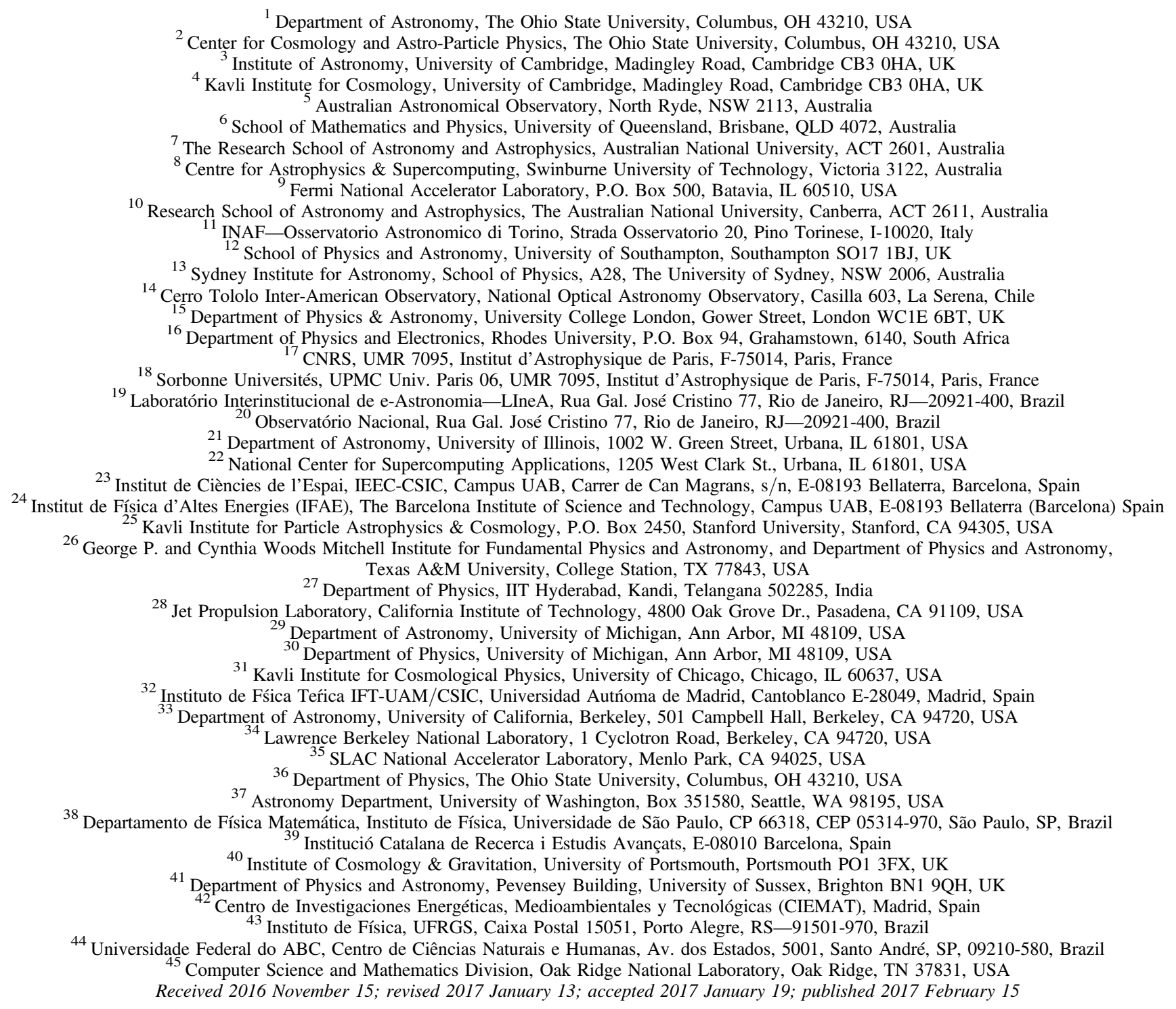

\begin{abstract}
We present a study of quasar selection using the supernova fields of the Dark Energy Survey (DES). We used a quasar catalog from an overlapping portion of the SDSS Stripe 82 region to quantify the completeness and efficiency of selection methods involving color, probabilistic modeling, variability, and combinations of color/ probabilistic modeling with variability. In all cases, we considered only objects that appear as point sources in the
\end{abstract}


DES images. We examine color selection methods based on the Wide-field Infrared Survey Explorer (WISE) midIR $W 1-W 2$ color, a mixture of WISE and DES colors $(g-i$ and $i-W 1)$, and a mixture of Vista Hemisphere Survey and DES colors $(g-i$ and $i-K)$. For probabilistic quasar selection, we used XDQSO, an algorithm that employs an empirical multi-wavelength flux model of quasars to assign quasar probabilities. Our variability selection uses the multi-band $\chi^{2}$-probability that sources are constant in the DES Year 1 griz-band light curves. The completeness and efficiency are calculated relative to an underlying sample of point sources that are detected in the required selection bands and pass our data quality and photometric error cuts. We conduct our analyses at two magnitude limits, $i<19.8 \mathrm{mag}$ and $i<22 \mathrm{mag}$. For the subset of sources with $W 1$ and $W 2$ detections, the $W 1-W 2$ color or $\mathrm{XDQSO}$ z method combined with variability gives the highest completenesses of $>85 \%$ for both $i$-band magnitude limits and efficiencies of $>80 \%$ to the bright limit and $>60 \%$ to the faint limit; however, the giW1 and $g i W 1+$ variability methods give the highest quasar surface densities. The XDQSOz method and combinations of $W 1 W 2 / g i W 1 / \mathrm{XDQSO} z$ with variability are among the better selection methods when both high completeness and high efficiency are desired. We also present the OzDES Quasar Catalog of 1263 spectroscopically confirmed quasars from three years of OzDES observation in the $30 \mathrm{deg}^{2}$ of the DES supernova fields. The catalog includes quasars with redshifts up to $z \sim 4$ and brighter than $i=22 \mathrm{mag}$, although the catalog is not complete up to this magnitude limit.

Key words: quasars: general

Supporting material: machine-readable table

\section{Introduction}

Quasars are highly energetic sources located at the centers of galaxies and are created by accretion of matter onto supermassive black holes. They are the most luminous subclass of active galactic nuclei (AGNs) and one of the most luminous classes of objects in the universe. Through accurate measurements of the quasar luminosity function and its evolution, the formation history of supermassive black holes can be studied in detail (Kelly \& Merloni 2012 and references therein). For studies of baryonic acoustic oscillations (BAO) and the Ly $\alpha$ forest, quasars act as tracers and backlights of matter clustering (e.g., Dawson et al. 2013; Font-Ribera et al. 2014; Delubac et al. 2015). All these studies benefit from efficient and/or complete selection of large quasar samples.

Sandage et al. (1965) pioneered quasar selection through the use of multicolor imaging data. Because quasars are not characterized by a single temperature like stars, they usually occupy different regions of color space. For instance, most quasars are bluer in the UV/visible and redder in the infrared. By virtue of this, color selection methods using UV, visible, and mid-infrared photometry are one of the easiest and most common ways to select quasars at various redshifts (e.g., Richards et al. 2002; Stern et al. 2012; Assef et al. 2013; Reed et al. 2015).

Nearly all quasars show $\sim 10 \%-20 \%$ stochastic variability at UV and visible wavelengths over timescales of many months to years (Koo et al. 1986; Hook et al. 1994; de Vries et al. 2003; Vanden Berk et al. 2004; Kelly et al. 2009; Kozłowski et al. 2010; MacLeod et al. 2010). Since only a small fraction of stars are variable at this level, and many of these variable stars are periodic, variability data can be used to separate quasars from stars. Models of variability such as a power law (Schmidt et al. 2010; Palanque-Delabrouille et al. 2011) or a damped random walk (MacLeod et al. 2010; Butler \& Bloom 2011; Ivezić \& MacLeod 2014) are usually used to identify the quasars. With the emergence of time-domain surveys such as Pan-STARRS (Kaiser et al. 2010) and the future Large Synoptic Survey Telescope (Ivezic et al. 2008), quasar selection based on variability will increase in significance and potentially help to fill the selection gaps in color selection techniques.
In addition to color and variability selection, more sophisticated selection methods have been developed, such as full multi-wavelength fitting of the spectral energy distribution (Chung et al. 2014), kernel density estimation (Richards et al. 2009), the likelihood method (Kirkpatrick et al. 2011a), neural networks (Yèche et al. 2010), and extreme deconvolution (Bovy et al. 2011a). These statistical methods model the underlying flux distribution of quasars based on empirical data and then assign probabilities that sources are quasars. The modeled phase space is expandable and can include variability in addition to flux. Statistical quasar selection methods have proven to be very efficient, as they incorporate multidimensional information on quasar properties (e.g., Ross et al. 2012).

We aim to quantify various quasar selection methods for luminous and point-source quasars such as color selection, probabilistic selection, variability selection, and combinations of the selection methods in the supernova fields of the Dark Energy Survey (DES). The DES is a $5000 \mathrm{deg}^{2}$ griz $Y$-band survey of the Southern sky designed to probe the nature of dark energy (Flaugher 2005; Frieman et al. 2013). DES includes 10 fields with a total area of $\sim 30 \mathrm{deg}^{2}$ that are surveyed at a higher cadence to search for Type Ia supernovae (SNe Ia), and are known as the DES supernova fields. We also present a catalog of spectroscopically confirmed quasars observed by OzDES (Yuan et al. 2015) in the DES supernova fields. OzDES is a complementary spectroscopic survey that targets the supernova fields to obtain redshifts for supernovae host galaxies and to conduct a quasar reverberation mapping experiment (King et al. 2015) and other projects.

The outline of the paper is as follows. In Section 2, we describe our photometric data sets and their corresponding surveys. These surveys include the DES, the Vista Hemisphere Survey (VHS; McMahon et al. 2013), and the Wide-field Infrared Survey Explorer (WISE) (Wright et al. 2010). We introduce the SDSS Stripe 82 quasar catalog from Peters et al. (2015) in Section 3, which we use to evaluate the quasar selection methods. In Section 4, we investigate selecting quasars as point sources. We evaluate the selection completeness and efficiency of various visible and IR color selection methods in Section 5. We analyze the XDQSOz probabilistic quasar selection algorithm (Bovy et al. 2011b, 2012; 
Table 1

DES Supernova Fields

\begin{tabular}{lccl}
\hline \hline Field Name & $\begin{array}{c}\text { R.A. } \\
\text { (h m s) }\end{array}$ & $\begin{array}{c}\text { Decl. } \\
\left({ }^{\circ} / \prime\right)\end{array}$ & Depth \\
\hline E1 & 003129.9 & -430034.6 & shallow \\
E2 & 003800.0 & -435952.8 & shallow \\
S1 & $0251 \quad 16.8$ & +000000.0 & shallow \\
S2 & 024446.7 & -005918.2 & shallow \\
C1 & 033705.8 & -270641.8 & shallow \\
C2 & 033705.8 & -290518.2 & shallow \\
C3 & 033035.6 & -280600.0 & deep \\
X1 & 021754.2 & -045546.2 & shallow \\
X2 & 022239.5 & -062443.6 & shallow \\
X3 & 022548.0 & -043600.0 & deep \\
\hline
\end{tabular}

DiPompeo et al. 2015) in Section 6. In Section 7, we consider a $\chi^{2}$-based variability selection method. We present the OzDES survey and the OzDES Quasar Catalog in Section 8. Finally, we close in Section 9 with discussions and conclusions. Throughout the paper, we adopted a flat $\Lambda$ CDM cosmology with $H_{0}=70 \mathrm{~km} \mathrm{~s}^{-1} \mathrm{Mpc}^{-1}$ and $\Omega_{0}=0.3$ (Komatsu et al. 2011). Unless stated otherwise, all visible magnitudes refer to the DES magnitudes. The AB magnitude system (Oke 1974) is used when quoting SDSS and DES magnitudes, while the Vega magnitude system (Johnson \& Morgan 1953) is used for WISE and VHS magnitudes.

\section{Photometry}

\subsection{Dark Energy Survey}

The DES is a wide-area $5000 \mathrm{deg}^{2}$ survey of the Southern Hemisphere in the grizY bands (Flaugher 2005; Frieman et al. 2013). Using the Dark Energy Camera (DECam; Flaugher et al. 2015) at the $4 \mathrm{~m}$ Blanco telescope at the Cerro Tololo Inter-American Observatory, DES aims to probe the nature of dark energy using four different astrophysical probes: SNe Ia, $\mathrm{BAO}$, galaxy clusters, and weak lensing. The planned $5 \sigma$ point source depths of the survey are $g=26.5 \mathrm{mag}, r=26 \mathrm{mag}$, $i=25.3 \mathrm{mag}, \quad z=24.7 \mathrm{mag}$, and $Y=23 \mathrm{mag}$ (Mohr et al. 2012). DES is covering a much larger area than other surveys of similar depth (e.g., the NOAO Deep Wide-field Survey, ${ }^{46}$ Jannuzi \& Dey 1999) and is much deeper than other surveys of larger area (e.g., SDSS and Pan-STARRS), so it is well suited to identifying new quasars. The survey finished its third season of operation in February 2016 (Diehl et al. 2016) and started its fourth observing season in August 2016.

DES conducts a multi-epoch supernova survey of two deep fields (C3 and X3) and eight shallow fields to search for $\mathrm{SNe}$ Ia (Bernstein et al. 2012). Each supernova field subtends $\sim 3 \mathrm{deg}^{2}$, and so $30 \mathrm{deg}^{2}$ in total. The supernova survey has a mean cadence of $\sim 7$ days in the griz bands. While the main DES survey is conducted under good seeing conditions (at a median FWHM $\sim 0$ ".9), it switches to imaging the supernova fields when the seeing increases to $\gtrsim 1$ !' 1 or when the supernova fields have not been observed for more than a week. The supernova component is expected to comprise roughly onethird or $\sim 1300 \mathrm{hr}$ of the total DES observing time. Table 1 shows the names and field centers of the 10 supernova fields. Most of the supernova fields are also well studied by other surveys such as the Chandra Deep Field-south (CDFS; Xue

\footnotetext{
46 http://www.noao.edu/noao/noaodeep/
}

et al. 2011) and the VIMOS-VLT Deep Survey (VVDS; Le Fèvre et al. 2005). These fields are optimal for studying variability-based quasar selection since they have more photometric epochs than the main DES wide-field survey. By the end of the five-year survey, all 10 supernova fields are expected to have more than 10 times the exposure time of the wide-field survey and more than 100 epochs, and to reach an expected $5 \sigma$ point-source depth in the griz bands of $\sim 28 \mathrm{mag}$ for the deep supernova fields and $\sim 26.5 \mathrm{mag}$ for the shallow fields (Bernstein et al. 2012).

For our work, we used the coadded catalog Y1A1_COADD_OBJECTS_DFULL for the DES year one (Y1, begun in 2013B) observations of the supernova fields. The catalog combines all available exposures (300 or more) from the DES Y1 operation and some from the Science Verification (SV) phase that have sufficient image quality (typical seeing FWHM $<1$ "! 1 , or 1 "' 25 in some cases). It is typically $\sim 2$ mag deeper than the DES Y1 wide-field coadded catalog. The catalog includes the weighted average of many photometric quantities from single-epoch DES data. For example, wavgcalib_mag_psf and wavg_magerr_psf are the weighted averages of the single-epoch mag_psf and magerr_psf values. The weighted averages are often found to be more accurate than the coadded quantities derived from SExtractor (Bertin \& Arnouts 1996), especially in areas where the number of epochs is small or where the coadded point-spread functions (PSFs) are not well fit by PSFEx (Bertin 2011). For the rest of this paper, we used the weighted average quantities whenever referring to DES photometry, such as wavgcalib_mag_psf_[grizY] and wavg_magerr_psf_[grizY] for the magnitudes and magnitude errors.

\subsection{Vista Hemisphere Survey}

The VHS (McMahon et al. 2013) ${ }^{47}$ is a wide-field nearinfrared (NIR) survey of the Southern Hemisphere to a depth 30 times deeper than 2MASS (Kleinmann et al. 1994) in the $J$ and $K$ bands. Some parts of the sky are also imaged in the $Y$ and $H$ bands. VHS aims to cover $18,000 \mathrm{deg}^{2}$ of the Southern Hemisphere and overlaps $\sim 4500 \mathrm{deg}^{2}$ of the DES footprint in the South Galactic Cap. It is the deepest NIR survey that overlaps a large fraction of the DES footprint. In the overlap region, VHS has a median $5 \sigma$ point source detection depth of $J_{\text {Vega }}=20.3 \mathrm{mag}$ and $K_{\mathrm{Vega}}=18.6 \mathrm{mag}$ with $80 \%$ completeness (Banerji et al. 2015). We used the publicly available Data Release 3 source/merged catalog ${ }^{48}$ vhs Source and the default point-source aperture-corrected magnitude and error, xAperMag3 and xAperMag3Err where $x$ refers to the desired band.

\subsection{WISE}

WISE (Wright et al. 2010) is an all-sky survey of the mid-IR sky at $3.4,4.6,12$, and $22 \mu \mathrm{m}(W 1, W 2, W 3$, and $W 4)$. After it depleted its hydrogen cryogen in 2010, the WISE mission was renamed NEOWISE (Mainzer et al. 2011) and it continued to survey the sky in the $W 1$ and $W 2$ bands until 2011. It was then reactivated in 2013 to conduct a "post-cryogenic" three-year survey of the sky in the $W 1$ and $W 2$ bands (Mainzer et al. 2014). At present, over $99 \%$ of the sky has $\geqslant 23$ exposures in these two bands.

\footnotetext{
47 http://www.vista-vhs.org/

48 http://horus.roe.ac.uk/vsa/
} 
We used the instrumental profile-fit photometry from the AllWISE source catalog 49,50 , i.e., wxmpro (magnitude), wxsigmpro (magnitude error), and wxsnr (signal-to-noise ratio) where $x$ is 1 or 2 , for the rest of this paper. The AllWISE catalog combines data from both the WISE cryogenic and the NEOWISE post-cryogenic phases, which improves the $W 1$ and W2 sensitivities. Magnitude limits for the ALLWISE catalog are $16.9 \mathrm{mag}$ and $16.0 \mathrm{mag}$ in $W 1$ and $W 2$, respectively, in the Vega system (Wright et al. 2010; Stern et al. 2012).

\section{Quasar Catalog of Peters et al. (2015)}

We used a subset of the SDSS Stripe 82 quasar catalog from Peters et al. (2015, hereafter referred to as P15) that overlaps with the DES supernova fields for our analyses of the quasar selection methods. P15 used a Bayesian analysis combining color and variability to identify 36,569 quasar candidates in the SDSS Stripe 82 field, of which 35,820 (98\%) are considered to be "good" quality candidates that pass their color cuts for removing stellar and white dwarf contaminants. Of these "good" candidates, 36\% are spectroscopically confirmed, and $92 \%$ of the spectroscopically confirmed quasars are brighter than coadded $i_{\text {SDSS }}=19.9$ mag. Using that sample, P15 estimated a (spectroscopic) completeness of $94.3 \%$ for the overall "good" quasar candidate sample at $i_{\text {SDSS }}=19.9$ mag (in agreement with Vanden Berk et al. 2005). The rest of the "good" candidates with no spectroscopic confirmation reach a depth of $i_{\text {SDSS }} \sim 22 \mathrm{mag}$; their redshifts are estimated using optical photometry and astrometry. P15 have limited spectroscopic data to measure the completeness and efficiency of their "good" candidates, including deeper spectroscopy for $\sim 5000$ quasars with $19<i_{\text {SDSS }}<22$ mag from the BOSS DR10 and DR12 quasar catalogs. The P15 "good" candidates recover $\sim 97 \%-98 \%$ of these spectroscopically confirmed quasars, so they conclude that their sample is $\sim 97 \%$ complete up to $i_{\text {SDSS }}<22$ mag. We conduct our quasar selection analyses using both the bright $\left(i_{\text {SDSS }}<19.9 \mathrm{mag}\right)$ and total $\left(i_{\text {SDSS }} \lesssim 22\right.$ mag) samples.

SDSS Stripe 82 has a $5.7 \mathrm{deg}^{2}$ overlap with the DES supernova fields in the $\mathrm{S} 1$ and $\mathrm{S} 2$ fields. For our selection analyses, we considered the $\mathrm{P} 15$ catalog only within this region of overlap and we call this subset the P15-S1S2 Quasar Catalog. Within the overlap region, the P15-S1S2 Quasar Catalog contains 975 quasar candidates. We cross-matched the P15-S1S2 Quasar Catalog with the DES Y1A1_COADD_OBJECTS_DFULL catalog (see Section 2.1) with a 0".5 matching radius, resulting in 900 matches (a larger matching radius of 1 !"0 returns two extra matches). From the 900 matches, 671 (120) have $i<22 \mathrm{mag}$ ( $i<19.8 \mathrm{mag}$ ), of which $60 \%(95 \%)$ have spectroscopic redshifts from the P15 catalog. Using the color terms in Section 7, we estimated $i_{\text {SDSS }}=19.9$ mag to be $i \sim 19.8$ mag.

We treated the P15-S1S2 catalog as a truth catalog for analyses of our selection methods at the bright $(i<19.8 \mathrm{mag}$ ) and faint $(i<22 \mathrm{mag})$ limits. We also defined a corresponding catalog of non-quasars in the overlapping DES S1 and S2 fields at both magnitude limits, which consists of point sources that are not in the P15-S1S2 Quasar Catalog. We define point sources in Section 4 and use the point source cut to reduce contamination from extended sources. We cross-matched the

\footnotetext{
49 http://wise2.ipac.caltech.edu/docs/release/allwise/

${ }^{50}$ http://irsa.ipac.caltech.edu/cgi-bin/Gator/nph-dd
}

P15-S1S2 catalog and the non-quasar catalog with VHS and WISE with matching radii of $0 . ! 5$ and 3 !" 0 , respectively (a larger matching radius of 1 !" 0 with VHS returns $\sim 1 \%$ extra matches and $\sim 80 \%$ of the matches with WISE are within $1 . " 0$ ). Figure 1 shows the distributions of spectroscopic redshift and absolute $i$-band magnitude for the matched total and bright samples of the P15-S1S2 Quasar Catalog, while Figure 2 shows their distributions of DES, WISE, and VHS magnitudes.

We imposed a set of preliminary cuts to define the underlying sample from which to calculate the completeness and efficiency of the selection methods in this work. Objects are required to be point sources, have DES flags_[griz] $<3$ in at least one of the griz bands (i.e., not close to a bright neighbor and not originally blended), have magnitude errors $<1 \mathrm{mag}$ in the gri bands (i.e., detected in those bands), and be detected in all the required non-DES selection bands. Figure 3 shows the fraction of the P15-S1S2 quasars that satisfy the detection cut (s) for each selection method as a function of $i$-band magnitude. There are 671 total (120 bright) P15-S1S2 quasars, of which 563 (105) satisfy the cuts in point source, flag, and magnitude error, where the flag cut removes $\sim 11 \%$ of the $i<22$ mag and $8 \%$ of the $i<19.8$ mag quasars. Of these 563 (105) quasars, 405 (103) have WISE W1 detections, 308 (101) have WISE W1 and $W 2$ detections, and 291 (104) have VHS $K$-band detections. Note that the median $K$-band depth in the P15$\mathrm{S} 1 \mathrm{~S} 2$ region is shallower than the median VHS depth over the wider area quoted in Banerji et al. (2015).

We give the completeness and efficiency for all the selection methods relative to sources that pass the preliminary cuts. Completeness is defined as the fraction of P15-S1S2 quasars that are selected, for the subset of the P15-S1S2 quasars that have sufficient data to apply the selection method. In other words, the numerator and denominator are only comprised of objects with sufficient data to apply the selection method. Efficiency is defined as the number of selected quasars divided by the total number of sources that satisfied the selection criterion. We will report the results for the selection methods for the full $i<22 \mathrm{mag}$ sample followed by the results for the bright sample $i<19.8$ mag in parentheses.

\section{Selection of Point Sources}

Shape selection can be used to separate most quasars from most galaxies, because quasars usually appear to be point sources and galaxies are usually resolved. This works well with the DES images because of their excellent image quality, except in a minority of cases where their superb depth detects the host galaxy. We used the DES weighted average of the SExtractor (Bertin \& Arnouts 1996) spread_model star/galaxy classifier, which is a discriminant between the best-fitting PSF model and a more extended model (Desai et al. 2012). Specifically, we used the cut $\mid$ wavg_spread_model_r $\mid<0.003$ + spreaderr_model_r to identify point sources (Drlica-Wagner et al. 2015).

We calculated the completeness of the shape selection criterion using a sample of point sources with very high confidence from regions of the Canada-France-Hawaii Telescope Lensing Survey (CFHTLenS; Heymans et al. 2012; Erben et al. 2013) that overlap three of the DES supernova fields (X1, X2, and X3). CFHTLenS combines five years' worth of data from the CFHT Legacy Survey and has reliable shape estimates calculated as part of the optimization for weak lensing analyses. We used the class_star and lensfit (Miller 


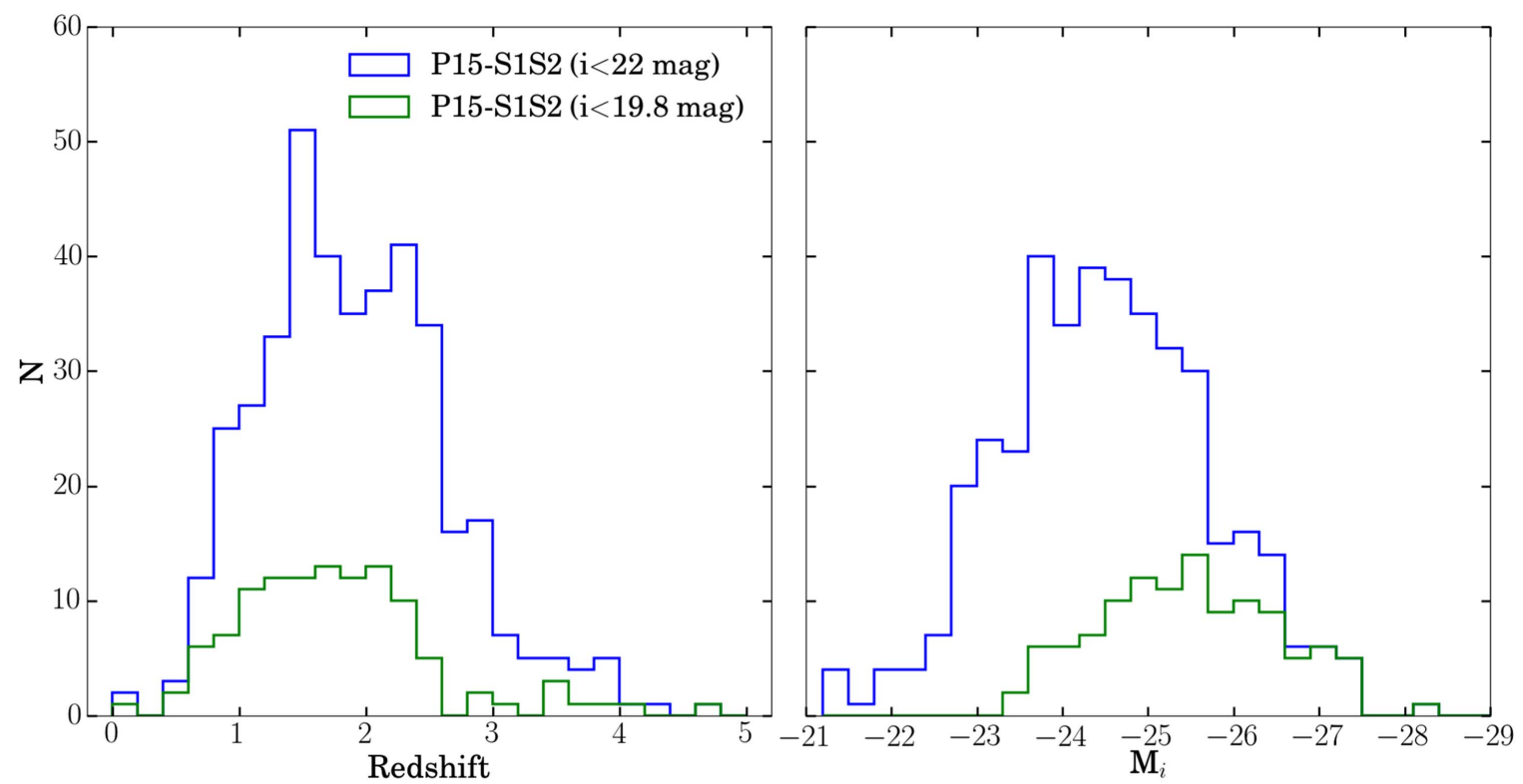

Figure 1. Distributions of spectroscopic redshifts and absolute $i$-band magnitudes for the bright and total samples of the P15-S1S2 Quasar Catalog with DES matches. The absolute magnitudes are calculated using DES $i$-band magnitudes and $k$-corrected spectroscopic redshifts from the left panel, with $k$-corrections from Richards et al. (2006).

et al. 2013) parameters from the public CFHTLenS catalog to select point sources, defined to be sources with class_star $>0.98$ or lensfit $=1$, and analyzed the three supernova fields separately, because the fields have different depths, with the X3 field being the deepest. We tested our shape selection on the P15-S1S2 Quasar Catalog. All CFHTLenS objects and P15S1S2 quasars are required to have DES flags_[griz] $<3$ in at least one of the griz bands to ensure the sources are not close to bright neighbors and not originally blended. Additionally, the sources need to have DES magnitude errors of $<1$ mag in the gri bands, where a magnitude error of $1 \mathrm{mag}$ corresponds to nondetection.

The left panel of Figure 4 shows the fraction of CFHTLenS point sources and P15-S1S2 quasars that pass our point-source selection criterion as a function of $i$-band magnitude in the three DES supernova X-fields. For selecting CFHTLenS stars, the completeness exceeds $90 \%$ for $i \lesssim 20.5 \mathrm{mag}$ and then drops to $\sim 80 \%$ by $i=22 \mathrm{mag}$. At $i>20 \mathrm{mag}$, the stellar sources from CFHTLenS are likely incomplete and the uncertainties in spreaderr_model_r are becoming large. The right panel of Figure 4 shows the wavg_spread_model_r $r$ shape information for the CFHTLenS stars and P15-S1S2 quasars as a function of $i$-band magnitude. The blue line illustrates the typical behavior of the shape cut as a function of magnitude using the median value for CFHTLenS stars in magnitude bins of $\Delta m=0.4 \mathrm{mag}$. It becomes more forgiving at fainter magnitudes due to the increase in spreaderr_model_r. The exact cut varies even for objects with the same apparent magnitude because of variations in the data such as the background and the number of coadd tiles. The shape cut selects quasars from the P15-S1S2 Quasar Catalog with a completeness of $99.6 \%$ for $i<22$ mag. We apply the shape cut in the selection analyses to select point sources.

\section{Color Selection}

Quasars have a nonthermal continuum with UV/optical spectra that can be approximated by a power law, in contrast to the single-temperature blackbody that roughly describes most stars. As a result, quasars tend to occupy distinct regions of color-color space, unlike stellar sources (Richards et al. 2002; Croom et al. 2004; Lacy et al. 2004; Maddox et al. 2012; Stern et al. 2012; Assef et al. 2013). Color cuts are thus commonly used to select quasars. Low-redshift $(z \lesssim 2)$ quasars are frequently selected using $u$-band data that identify the bright ultraviolet continuum emission of quasars. At $z>2.2$, the $\mathrm{Ly} \alpha$ line exits the $u$-band and enters the bluer $b$ or $g$ optical bands, resulting in redder $u-b / g$ colors that are similar to the color of stars. Although the wide-field region of DES lacks the $u$ band, many of the most interesting problems require finding quasars at higher redshifts where this selection approach fails. We focus on combinations of optical, near-IR, and mid-IR data that can effectively identify both high- and low-redshift quasars even in the absence of $u$-band data.

We evaluate three color selection methods, $W 1-W 2, g-i$ versus $i-W 1$, and $g-i$ versus $i-K$, using the P15-S1S2 Quasar Catalog. The $g i W 1$ and $g i K$ color selections were initially used or developed to target AGNs for the OzDES reverberation mapping monitoring campaign and they were designed based on the stellar locus to reduce contamination from stars.

\subsection{WISE W1 - W2 Color Selection}

Quasars generally have a red $W 1-W 2$ color due to emission from hot dust (in the case of low-redshift quasars) or the accretion disk (in the case of high-redshift quasars), unless a strong emission line happens to enter the $W 1$ band (Assef et al. 2010). Contaminants with similarly red $W 1-W 2$ colors are cool brown dwarfs and dusty stars. For example, brown dwarfs with spectral class cooler than T1 have red colors in these WISE bands due to methane absorption (Cushing et al. 2011; Kirkpatrick et al. 2011b). We investigated quasar selection using $W 1-W 2>0.7$ (Vega magnitudes) based on Stern et al. (2012). The sample is limited to point sources with a 

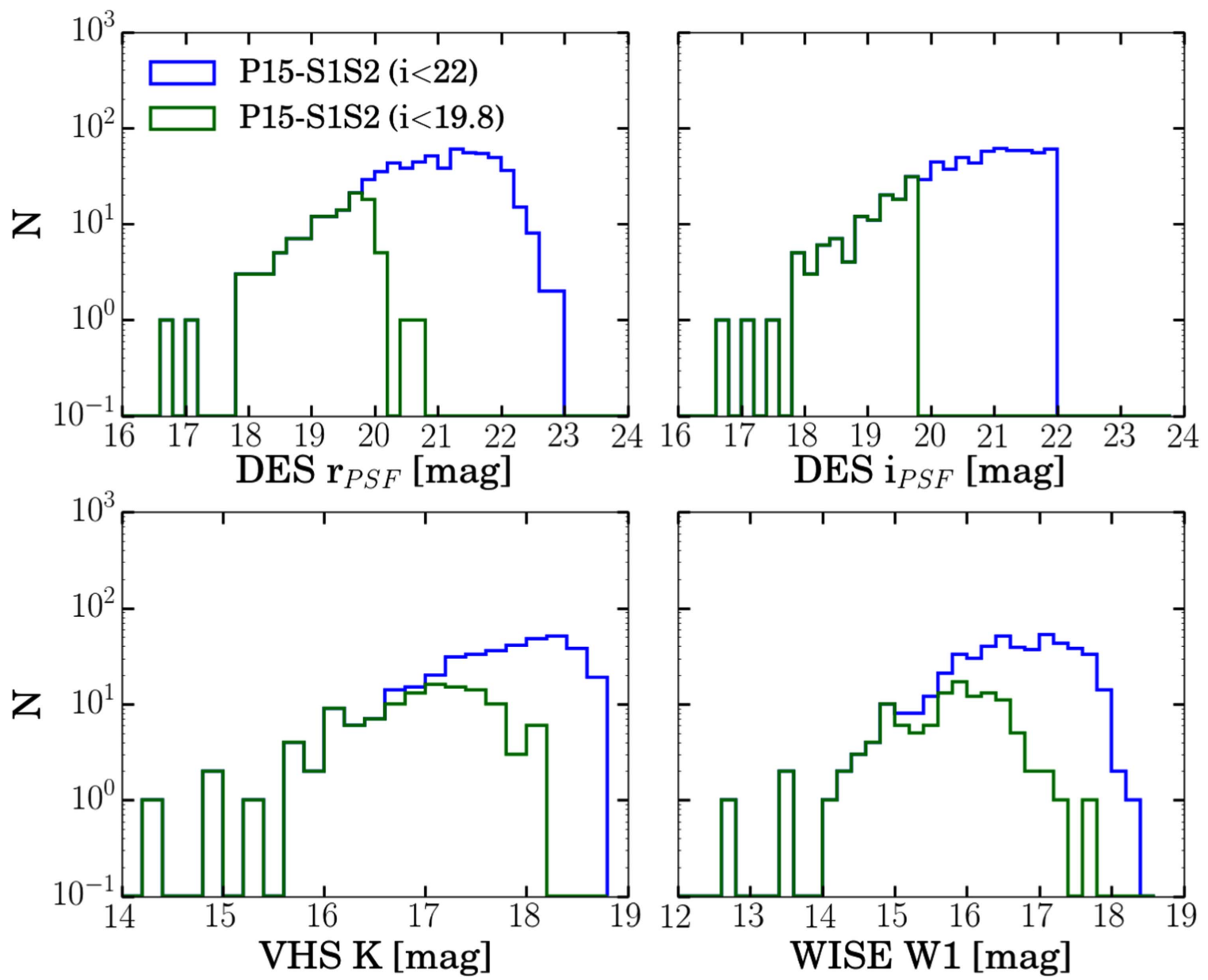

Figure 2. Distribution of the P15-S1S2 quasars with DES matches in the visible to the mid-IR wavelengths.

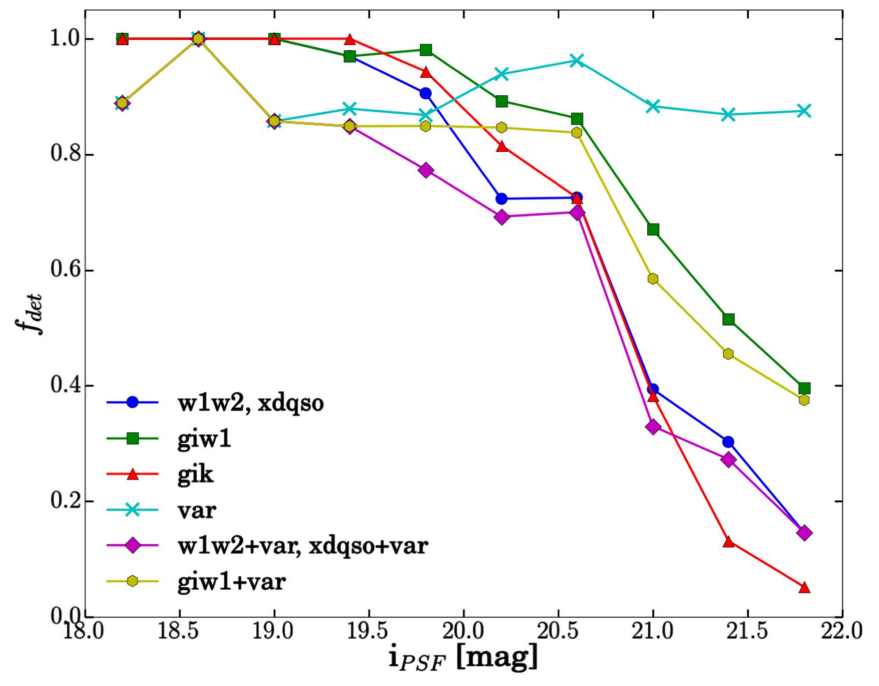

Figure 3. The fraction $f_{\text {det }}$ of P15-S1S2 quasars that meet the selectiondependent detection threshold(s) relative to the quasars that satisfy the cuts in flag, magnitude error, and point source. The lines are color-coded according to the type of selection and illustrate the consequences of requiring WISE, VHS, or epoch limits on the sample size. The $W 1 W 2$ and XDQSO methods have the same $f_{\text {det }}$ curve because they both require detections in the $W 1$ and $W 2$ bands.

signal-to-noise ratio of $\mathrm{S} / \mathrm{N}>5$ in both the $W 1(\sim 16.9 \mathrm{mag})$ and $W 2(\sim 16 \mathrm{mag})$ bands.

We calculate the completeness and efficiency of this color selection method relative to the total and bright samples of the
P15-S1S2 Quasar Catalog. In each case we give the results for the $i<22 \mathrm{mag}$ sample followed by the results for the $i<19.8 \mathrm{mag}$ sample in parenthesis. This color selection identifies 287 (92) of the 308 (101) P15-S1S2 quasars with the data necessary to apply this cut, for a completeness of $93 \%$ $(91 \%)$. There are also 246 (37) other point sources that satisfy this color selection, for an efficiency of 54\% (71\%). Figure 5 shows the $W 1-W 2$ colors as a function of redshift and apparent $i$-band magnitude for the P15-S1S2 quasars and the point-source non-quasars in the DES S1 and S2 fields.

\section{2. giW1 Selection}

Quasars are expected to be bluer in the $g$-band than in redder optical filters, but red when compared to the mid-IR (Wu et al. 2012; Myers et al. 2015; Chehade et al. 2016). We therefore investigated a color selection method combining

$$
\begin{aligned}
& g-i<1 \text { with } \\
& \quad g-i<1.195\left(i-W 1_{\mathrm{AB}}\right)+1.317
\end{aligned}
$$

where all the magnitudes are on the $\mathrm{AB}$ system, with $W 1_{A B}=W 1_{\text {Vega }}+2.699 .{ }^{51}$ The $g-i$ limit is intended to reduce contamination from compact galaxies, but excludes high-redshift quasars with $z>3.5$. This color selection identifies 369 (90) of the 405 (103) P15-S1S2 quasars with

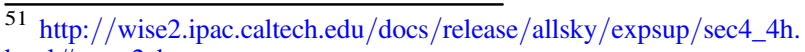
html\#conv2ab
} 

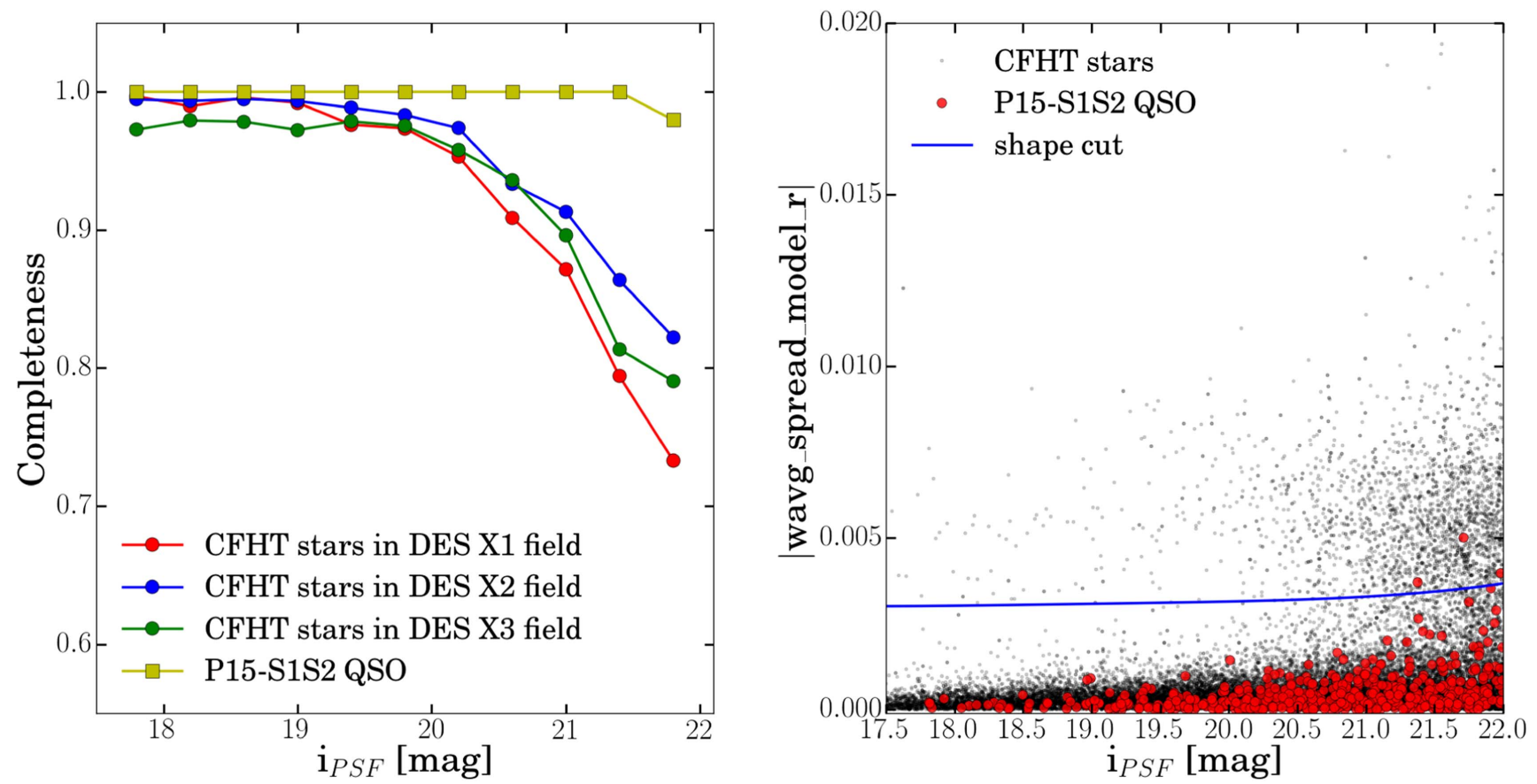

Figure 4. Left panel: fraction of objects classified as stars by CFHTLenS that are also classified as point sources by the cut $\mid$ wavg_spread_model_r $\mid<0.003+$ spreaderr_model_ $r$ as a function of DES $i$-band magnitude. Most of the P15-S1S2 quasars satisfy this shape cut, with only $0.4 \%$ failing at $i<22$ mag, reaching a completeness of $99.6 \%$. Right panel: shape information for the CFHTLenS point sources and quasars from the P15-S1S2 Quasar Catalog as a function of $i$-band magnitude. Gray points are the CFHTLenS point sources while red points are the P15-S1S2 quasars. The blue line is a representation of the shape cut that illustrates how it becomes more lenient at fainter magnitudes due to the increase in spreaderr_model_r.

the data necessary to apply this cut, for a completeness of $91 \%$ (87\%). There are 391 (58) other point sources that satisfy this color selection, for an efficiency of 49\% (61\%). Figure 6 shows the $i-W 1$ and $g-i$ colors for quasars in the P15-S1S2 catalog and the point-source non-quasars in the S1 and S2 fields.

\section{3. giK Selection}

Quasars in the range $2<z<3$ have visible-wavelength colors similar to stars, making it challenging to select quasars in this redshift range with high efficiency using color selection. The traditional method to separate such intermediate-redshift quasars from stars is the UV excess method (Richards et al. 2002), because quasars at these redshifts have more UV flux than stars owing to the presence of the $\operatorname{Ly} \alpha$ emission line in the UV filters. Here, an alternative approach is needed because DES lacks $u$-band data. Intermediate-redshift quasars also have a NIR $K$-band excess compared to stars (Warren et al. 2000), so we tested the color cuts

$$
\begin{aligned}
& g-i<1.5 \text { with } \\
& g-i<1.152\left(i-K_{\text {Vega }}\right)-1.4, \text { with } K_{\text {Vega }}<18.6
\end{aligned}
$$

following Banerji et al. (2015). Note that this mixes DES AB with VHS Vega magnitudes. Similarly to Section 5.2, the $g-i$ limit, which is more relaxed here, is used to minimize contamination from compact galaxies but also excludes highredshift quasars $(z>3.5)$. The median $K$-band depth in the P15-S1S2 region is also shallower than the median VHS depth over the wider DES-VHS area quoted in Banerji et al. (2015). This color selection identifies 269 (93) of the 291 (104) P15S1S2 quasars with the data necessary to apply this cut, for a completeness of $92 \%$ (89\%). There are 344 (55) other point sources that satisfy this color selection, for an efficiency of $44 \%$ (63\%). Figure 7 shows the $i-K_{\mathrm{Vega}}$ and $g-i$ colors for quasars in the P15-S1S2 catalog and other point-source nonquasars in the S1 and S2 fields.

\section{XDQSO Selection}

The change in the observed colors of quasars with redshift restricts the effectiveness of any one set of colors in selecting quasars to a limited redshift range. Probabilistic techniques have been devised to select quasars more effectively over a broader range of redshifts based on empirical models of quasar and stellar photometry. One such technique is XDQSO (Bovy et al. 2011b), which uses density estimation in flux space to assign a quasar probability. The density estimation is based on the extreme deconvolution (XD) method (Bovy et al. 2011a), which rigorously infers distribution functions in the presence of low signal-to-noise and heterogeneous data. XDQSO was developed with a stellar training set from the SDSS Stripe 82 (Abazajian et al. 2009) and a quasar training set from the SDSS DR 7 quasar catalog (Schneider et al. 2010). Bovy et al. (2011b) applied XDQSO to the SDSS Data Release 8 (Aihara et al. 2011) to create an input quasar catalog for the BOSS survey (Ross et al. 2012). They found that XDQSO performs as well as color-based quasar selection methods at low redshift $(z<2.2)$, and better than all other color-based quasar selection methods at intermediate redshifts $(2.2 \leqslant z \leqslant 3.5)$.

We used the more recent $\mathrm{XDQSOz}$ implementation (Bovy et al. 2012), which has also been adopted by the extended-BOSS (eBOSS) survey (Myers et al. 2015). We applied XDQSOz by combining the DES griz magnitudes corrected for Galactic extinction (Schlafly \& Finkbeiner 2011) and WISE photometry. We transformed the DES magnitudes to the SDSS systems using 

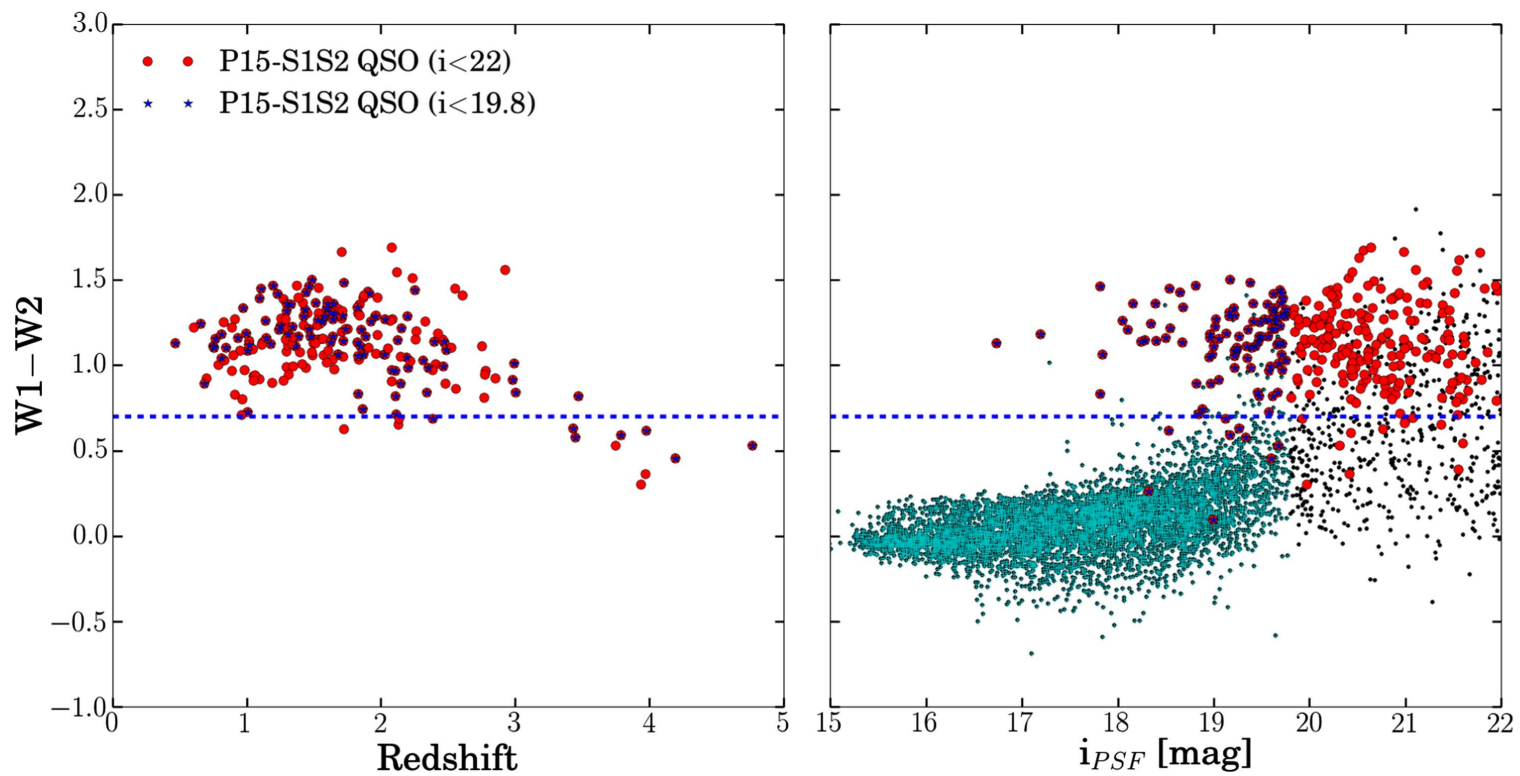

Figure 5. Left panel: WISE W1 - W2 color as a function redshift for quasars in the P15-S1S2 Quasar Catalog. Right panel: WISE color as a function of the DES $i$ band magnitude for the same quasars plus point-source non-quasars in the DES S1 and S2 fields, where black (cyan) points have $i<22 \mathrm{mag}(i<19.8 \mathrm{mag}$ ). The WISE color cut is shown by the dotted blue line in both panels. The WISE color is on the Vega system and the $i$-band magnitude is on the AB system.

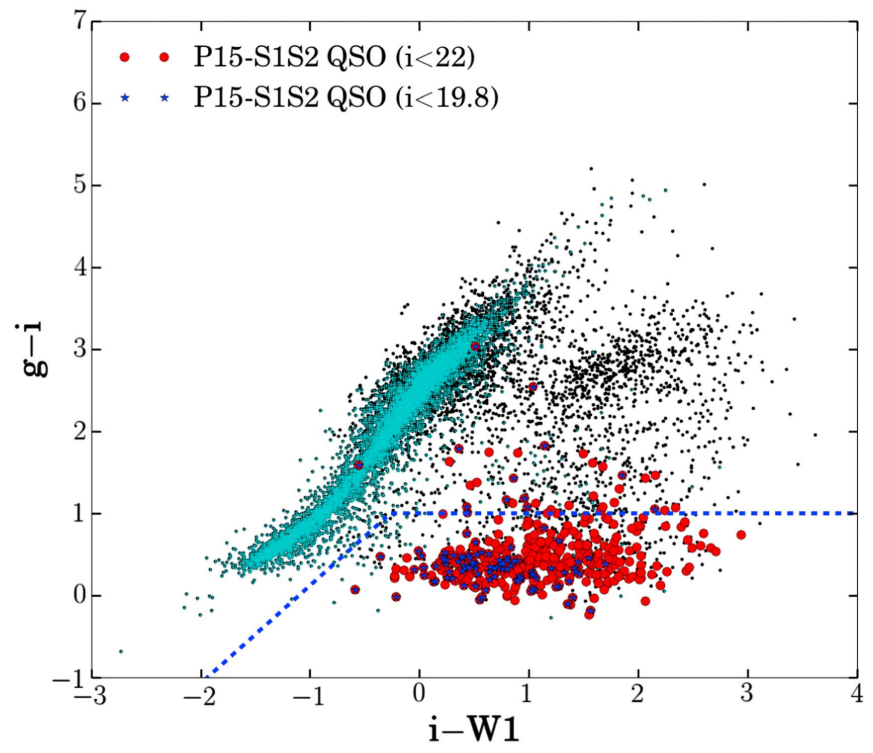

Figure 6. Color-color diagram of quasars from the P15-S1S2 catalog and point-source non-quasars in the DES S1 and S2 fields, where black (cyan) points are point sources with $i<22 \mathrm{mag}(i<19.8 \mathrm{mag})$. The blue dotted lines define the selection region. Note that the visible and WISE magnitudes are both in the $\mathrm{AB}$ system.

color corrections of

$$
\begin{aligned}
g_{\mathrm{DES}}-g_{\mathrm{SDSS}} & =-0.083(g-r)_{\mathrm{DES}}-0.024, \\
r_{\mathrm{DES}}-r_{\mathrm{SDSS}} & =-0.083(g-r)_{\mathrm{DES}}-0.004, \\
i_{\mathrm{DES}}-i_{\mathrm{SDSS}} & =-0.352(i-z)_{\mathrm{DES}}+0.017, \text { and } \\
z_{\mathrm{DES}}-z_{\mathrm{SDSS}} & =-0.104(i-z)_{\mathrm{DES}}-0.007
\end{aligned}
$$

derived from bright SDSS stars. ${ }^{52}$ Since XDQSOz expects a $u$ band measurement, we supplied a very small flux and an

\footnotetext{
52 We used objects from SDSS PhotoPrimary table that are between 17 and $19 \mathrm{mag}$, have errors less than $0.5 \mathrm{mag}$, are located in DES S1 and S2 fields, and have SDSS flags satisfying ! deblend_too_many_peaks \&\&!moved \&\& binned1 \&\&! satur_center \&\&!bad_counts_error \&\&! notchecked_center $\& \& !$ edge $\& \&$ psf_flux_interp.
}

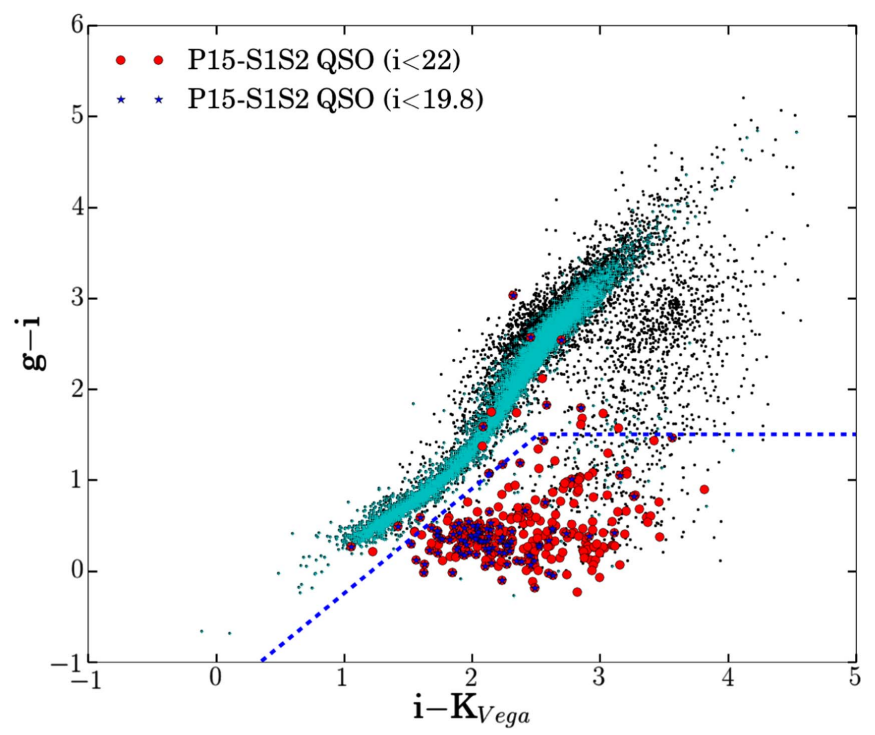

Figure 7. Color-color diagram of quasars from the P15-S1S2 catalog and point-source non-quasars in the DES S1 and S2 fields. Black (cyan) points are point sources with $i<22 \mathrm{mag}(i<19.8 \mathrm{mag})$. The blue dotted lines define the color selection region. The visible magnitudes are on the $\mathrm{AB}$ system and the VHS magnitude is on the Vega system.

inverse variance of $10^{-10}$ for this band. We found that including the near-IR data from VHS leads to a slight improvement, but at the expense of a much smaller sample because many sources lack the necessary VHS data. Figures 8 shows the $\mathrm{XDQSO}$ z probability distributions for the P15-S1S2 quasars and point-source non-quasars in the total and bright samples. The $\mathrm{XDQSO}$ z selection with a probability cut at $P_{\mathrm{QSO}}=0.5$ identifies 276 (93) of the 308 (101) P15-S1S2 quasars with the data necessary to apply this cut, for a completeness of 90\% (92\%). There are 185 (34) other point sources that satisfy this color selection, for an efficiency of $60 \%(73 \%)$. 

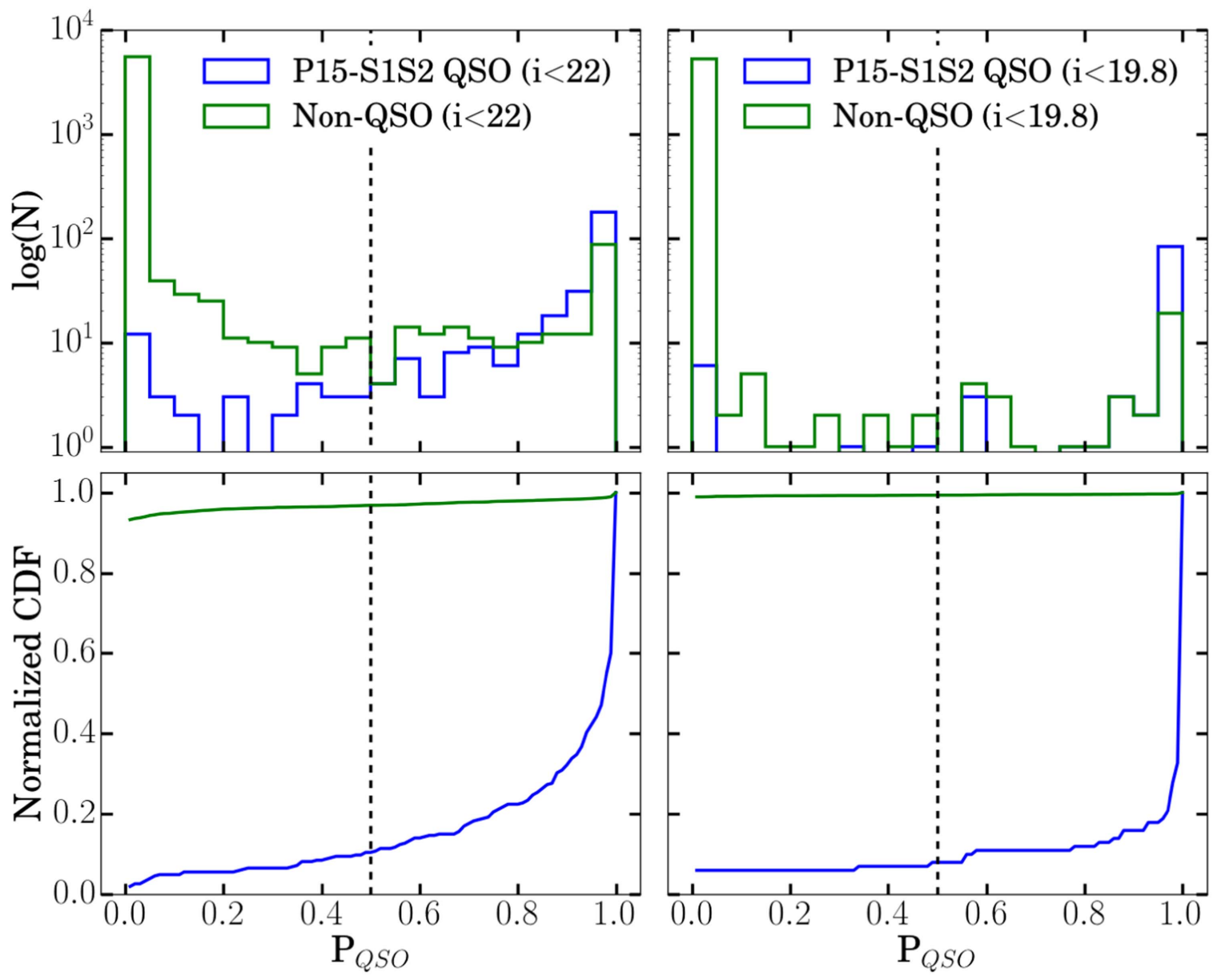

Figure 8. Top panel: distribution of $\mathrm{XDQSO}$ z quasar probabilities for the P15-S1S2 quasars and point-source non-quasars with WISE photometry at the faint and bright magnitude limits. The dotted line denotes the probability cut at $P_{\mathrm{QSO}}=0.5$. Bottom panel: the cumulative distribution of the same quasars and non-quasars in $P_{\mathrm{QSO}}$. The majority $(>90 \%)$ of the non-quasars have $P_{\mathrm{QSO}}<0.5$ while quasars mostly have $P_{\mathrm{Qso}}>0.5$.

\section{Variability Selection}

We obtained light curves from the DES Y1 single-epoch catalogs, Y1A1_IMAGE and Y1A1_FINALCUT, which span less than a year and typically have $\sim 15$ epochs. Because of the relatively limited temporal extent of these data, we cannot adopt sophisticated variability models based on fitting structure functions. Instead, we used a multi-band griz $\chi^{2}$ variability statistic to distinguish between variable and nonvariable sources. We calculated $\chi^{2}=\sum_{K=g, r, i, z} \sum_{j=1}^{N_{K}}\left(m_{K, j}-\left\langle m_{K}\right\rangle\right)^{2} / \sigma_{K, j}^{2}$ for each source and evaluated the null hypothesis that the source has constant magnitude $\left\langle m_{K}\right\rangle$ over $N_{K}$ epochs, where $\left\langle m_{K}\right\rangle$ is defined as the error-weighted mean magnitude $\left\langle m_{K}\right\rangle=$ $\left(\sum m_{K, j} / \sigma_{K, j}^{2}\right) /\left(\sum 1 / \sigma_{K, j}^{2}\right)$ for each band $K$. The single-epoch photometry for DES is limited by systematics rather than photon statistics. At $i<22 \mathrm{mag}$, the typical median single-epoch errors are 0.02 mag in $g, r, i$, and $z$ bands, which are comparable to the coadded DES errors in these bands. At $i<19.8 \mathrm{mag}$, the typical median single-epoch errors are 0.01, 0.004, 0.003, and 0.003 mag in griz, which are roughly half the typical median coadded errors. To account for the underestimated single-epoch errors, we imposed a minimum error floor of $\sigma_{j}=0.01 \mathrm{mag}$ in griz bands for the variability measurements.

Our variability criterion uses the chi-squared integrated probability $P\left(X_{\nu}^{2} \geqslant \chi^{2}\right)$ to reject the null hypothesis, where $\nu=\sum N_{k}-\sum k$ is the number of degrees of freedom and $X_{\nu}^{2}$ is the chi-squared distribution with $\nu$ degrees of freedom. The sources are required to have $N_{k} \geqslant 3$ and $\sum N_{k} \geqslant 6$. In other words, a source needs to have at least six epochs of data if only one band is available or at least three epochs of data in at least two bands. After the application of the shape, flag, and photometric cuts, 503 (90) quasars and 17,539 (6656) other point sources satisfied this condition at $i<22 \mathrm{mag}$ $(i<19.8 \mathrm{mag})$. Figure 9 shows their reduced- $\chi^{2}$ distributions.

We chose a threshold $P\left(X_{\nu}^{2} \geqslant \chi^{2}\right)$ value of 0.01 as our variability selection cut, which corresponds to rejection of the null hypothesis with 99\% significance. There are 444 (77) quasars and 3418 (799) non-quasars that pass the variability selection criterion with $P\left(X_{\nu}^{2} \geqslant \chi^{2}\right)<0.01$, resulting in an efficiency of $11.4 \%(8.8 \%)$ and a completeness of $88 \%$ $(86 \%)$. Figure 10 shows the efficiency and completeness as a function of the $P$-value for the P15-S1S2 quasars. The low efficiencies are likely due to the short baseline of our current light curves combined with contamination from stars that have a higher surface density and show low-level variability. Since our variability selection is simply based on the $\chi^{2}$ bound for fitting a constant magnitude, any sufficiently variable source will pass the cut. More sophisticated statistical models require longer observational baselines but can differentiate between quasar and stellar variability. The average number of epochs in the present variability sample is $\sim 13$ in gri bands and $\sim 40$ in the $z$ band spread over only half 


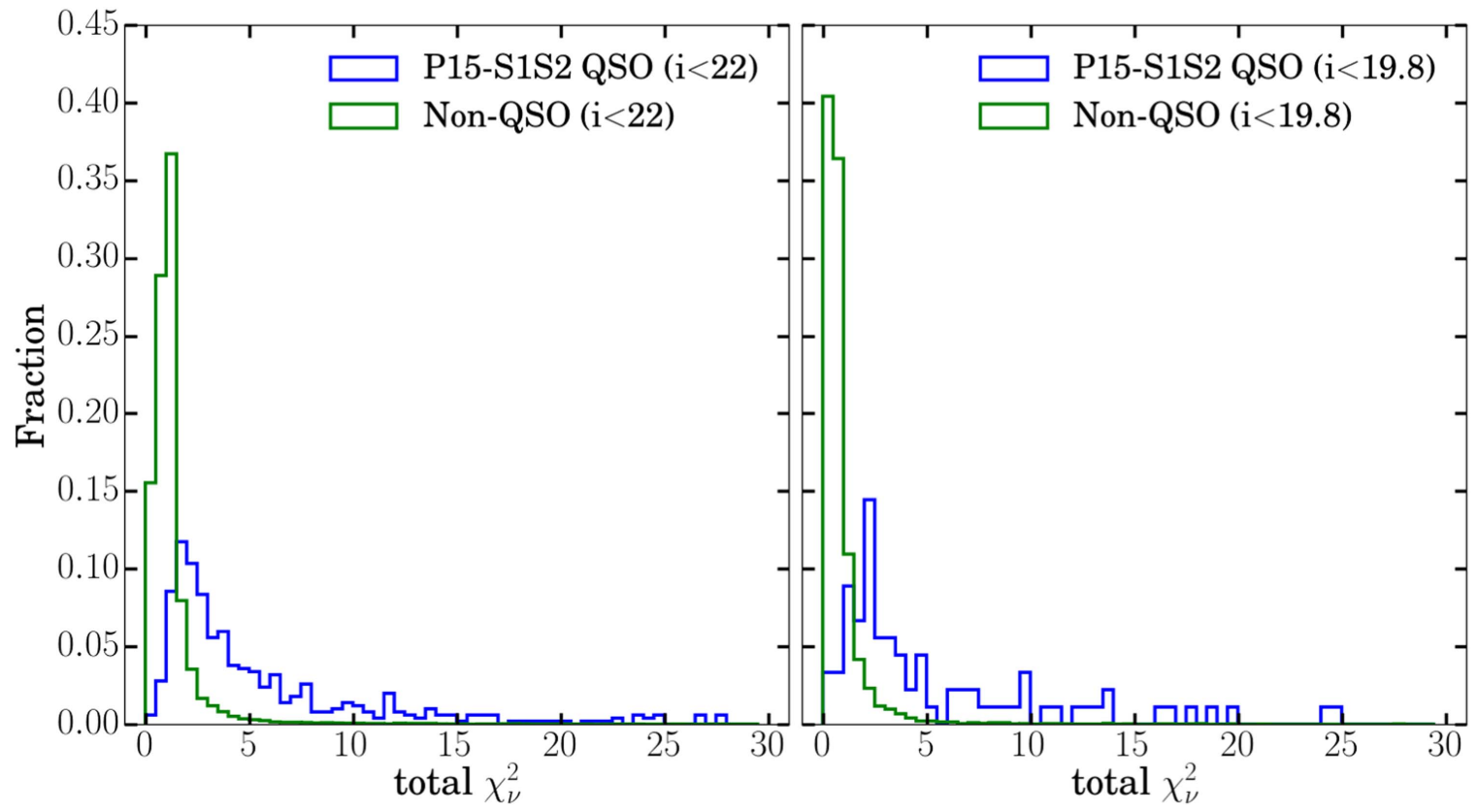

Figure 9. Reduced- $\chi^{2}$ distribution over DES bands with enough epochs for the P15-S1S2 quasars and point-source non-quasars at the faint and bright magnitude limits. While the non-quasars are well fit by a constant-magnitude model, the quasars show larger deviations from such a model.

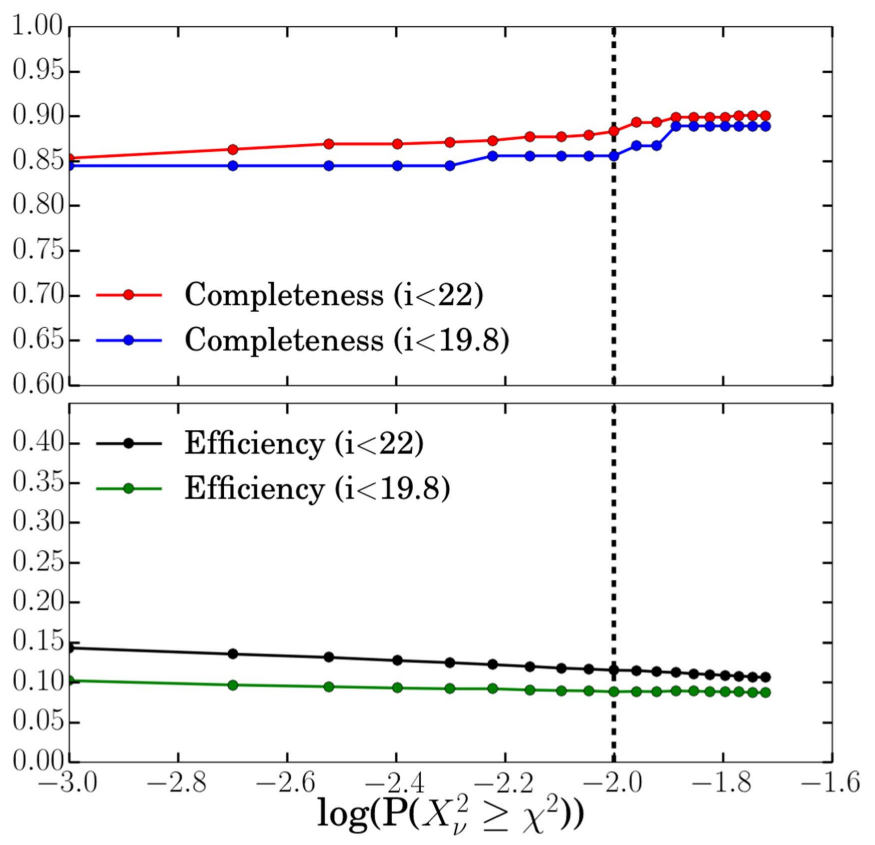

Figure 10. Completeness and efficiency of variability selection $P\left(X_{\nu}^{2} \geqslant \chi^{2}\right)>0.01$ for the P15-S1S2 Quasar Catalog, where $P\left(X_{\nu}^{2} \geqslant \chi^{2}\right)$ is the probability of rejecting the null hypothesis that sources are nonvariable. The dotted line at $\log \left(P\left(X_{\nu}^{2} \geqslant \chi^{2}\right)\right)=-2.00$ corresponds to rejecting the null hypothesis at $99 \%$ confidence.

a year. This is much smaller than the $\sim 60$ epochs spanning about six years in the SDSS Stripe 82 region (Sesar et al. 2007; Bramich et al. 2008). With the full five-year DES data, the number of epochs will increase by a factor of 5 and the probability that a quasar has varied significantly will increase due to the longer time baseline. This will improve the performance of the variability selection, as well as enabling the use of more sophisticated quasar variability models.
We investigated the performance of combinations of $W 1 W 2$ color, giW1 color selection, and XDQSOz methods with variability. The combinations $W 1 W 2+$ variability, giW1 +variability, and XDQSOz+variability give completenesses of $88 \%(81 \%), 86 \%(78 \%)$, and $85 \%(83 \%)$ and efficiencies of $63 \%(83 \%), 60 \%(80 \%)$, and $68 \%(83 \%)$, respectively, for the total (bright) sample. Compared to color selection, $\mathrm{XDQSOz}$ selection, or variability selection alone, these combinations produce higher selection efficiency due to fewer false positives, with a slight reduction in completeness (see Table 3).

\section{The OzDES Quasar Catalog}

The Australian Dark Energy Survey (OzDES; Yuan et al. 2015) is a spectroscopic survey of the DES supernova fields using the AAOmega spectrograph (Smith et al. 2004) on the Anglo-Australian Telescope (AAT). The field of view of the AAT multi-object fiber-positioning system (Lewis et al. 2002) is well matched to DECam, making the AAT a wellsuited spectroscopic follow-up instrument for DES.

OzDES commenced observations at the same time as DES in 2013B and aims to measure redshifts for thousands of host galaxies of Type Ia supernovae and black hole masses for hundreds of AGNs and quasars (King et al. 2015) using reverberation mapping (Blandford \& McKee 1982; Peterson 1993). Objects that range in brightness from $m_{r} \sim 17$ mag to $m_{r} \sim 25 \mathrm{mag}$ are selected for spectroscopy via a variety of criteria, such as supernova candidates, quasar candidates, galaxy cluster members, and photometric redshift calibration candidates (see Yuan et al. 2015 for further details). Under most circumstances, an object is observed repeatedly until a redshift is obtained. The observing targets and results of OzDES observations are compiled into a spectroscopic catalog known as the Global Redshift Catalog (GRC), which also includes redshifts from other spectroscopic surveys such as $6 \mathrm{dF}$ (Jones et al. 2004), SDSS (York et al. 2000), and VVDS (Le Fèvre et al. 2005). The GRC is updated after every OzDES 
Table 2

OzDES Quasar Catalog

\begin{tabular}{|c|c|}
\hline Column Name & Description \\
\hline ID & OzDES Global Redshift Catalog (GRC) ID \\
\hline DES coadd_objects_id & DES ID from the Y1A1_COADD_OBJECTS_DFULL catalog \\
\hline RA, DEC & R.A., Decl. \\
\hline ABS_I_MAG & Absolute $i$-band magnitude calculated using the OzDES redshift \\
\hline DES wavgcalib_mag_psf_[g, r, i, z, Y] & DES magnitudes (AB) (see Section 2.1) \\
\hline DES wavg_mag_err_[g, r, i, z, Y] & DES magnitude errors \\
\hline DES wavg_spreaderr_model_[g, r, i, z, Y] & DES spread_model errors \\
\hline WISE w1mpro, w2mpro & WISE magnitudes (Vega) from the ALLWISE catalog (see Section 2.3) \\
\hline WISE w1sigmpro, w2sigmpro & WISE magnitude errors \\
\hline VHS [y, j, h, ks]AperMag3 & VHS magnitudes (Vega) from the DR3 vhsSource catalog (see Section 2.2) \\
\hline VHS [y, j, h, ks]AperMag3Err & VHS magnitude errors \\
\hline \multirow[t]{6}{*}{ OzDES Selection Code } & 0 : Not targeted as a quasar candidate \\
\hline & 16: NED quasars \\
\hline & 32: giW1 selection (see Section 5.2) \\
\hline & 64: Similar selection to code 8 but with a relaxed blue cut in $g i K$ \\
\hline & 128: High redshift selection using gri and riz colors \\
\hline & 256: $u$-band selection \\
\hline
\end{tabular}

Note. Quasars with nondetections in DES bands, no WISE matches, or no VHS matches have entries of 99 for their magnitudes and errors. The naming schemes for the photometry follow the catalogs from which they are derived; see the relevant sections as indicated for more information. There are 44 columns in the catalog. (This table is available in its entirety in machine-readable form.)

observing season. In this work, we used the 2016 February version of the OzDES GRC.

Here we present the OzDES Quasar Catalog of 1263 OzDES sources with $M_{i}<-22 \mathrm{mag}$ and $i<22 \mathrm{mag}$ that are spectroscopically confirmed quasars. We constructed the catalog as follows. As the GRC does not provide photometric information, we cross-matched the GRC with the DES supernova field catalog Y1A1_COADD_OBJECTS_DFULL (see Section 2.1). Sources within 1.1 of the 10 supernova field centers (see Table 1) and with $i<22 \mathrm{mag}$ are matched using a matching radius of 0.15 . We used $k$-corrections from Richards et al. (2006) and the astropy cosmology package ${ }^{53}$ to calculate the absolute $i$-band magnitude $M_{i}$ of the OzDES sources for a flat $\Lambda \mathrm{CDM}$ cosmology with $H_{0}=70 \mathrm{~km} \mathrm{~s}^{-1} \mathrm{Mpc}^{-1}$ and $\Omega_{0}=0.3$. Specifically, as the $k$-corrections in Richards et al. (2006) are for $\mathrm{z}=2$, we applied the offset $M_{i}(z=0)=M_{i}(z=2)+0.596$ in accordance with Equation (1) of Richards et al. (2006). We narrowed down the sample to sources with "good" OzDES redshifts (quality flags 3 or 4 in the GRC). Finally, we visually inspected the spectra of sources that have $M_{i}<-22$ mag, which are roughly 2200 objects in total. We looked for the presence of broad emission lines, such as Ly $\alpha \lambda 1216, \mathrm{C}$ IV $\lambda 1548, \mathrm{C}$ III $\lambda 1909$, and $\mathrm{Mg}$ II $\lambda 2798$, and ensured that the line identifications are consistent with the OzDES redshifts. For sources with no clear lines ( $\sim 40 \%$ of the visually inspected sources), we re-examined their spectra and redshifts using the OzDES redshifting software MARZ (Hinton et al. 2016). Only sources that passed our visual inspection are included in the catalog.

\footnotetext{
53 http://www.astropy.org
}

The OzDES Quasar Catalog consists of 1263 quasars brighter than $i=22 \mathrm{mag}$, corresponding to a quasar surface density of $\sim 42 \mathrm{deg}^{-2}$. It includes many previously known quasars. The new quasars discovered by OzDES include a recently discovered post-starburst quasar with broad absorption lines at $z=0.65$ (see Mudd et al. 2016). Multiwavelength photometry from DES, VHS, and WISE is provided as part of the catalog. Table 2 describes the catalog, and the full table is available in the online journal. Figure 11 shows the absolute $i$-band magnitude and redshift distributions of the quasars.

The OzDES Quasar Catalog is neither homogeneously selected nor complete. The OzDES selection codes in Table 2 note how the quasars were initially selected for OzDES observations. For instance, the selection codes 1, 2, and 16 refer to previously known quasars from DES SV observations (Banerji et al. 2015), the VVDS survey, and NED, ${ }^{54}$ respectively, while quasars with selection code 0 are mostly identified as transients and DES photo- $z$ calibration targets. Selection code 4 refers to objects that were best matched to a quasar template using the LePhare photo- $z$ estimation tool (Arnouts et al. 1999). Target selection with LePhare only used the stellar and quasar templates, because the input catalog is limited to point-like sources. Selection codes 8, 32, and 64 are color-selected quasars using the color cuts described in Sections 5.2 and 5.3 for sources that satisfied a shape cut. Finally, quasars with selection codes 128 and 256 are selected using color cuts informed from SDSS (Richards et al. 2002): $g-r$ versus $r-i$ and $r-i$ versus $i-z$ in the case of code 128,

${ }^{54}$ https://ned.ipac.caltech.edu/ 

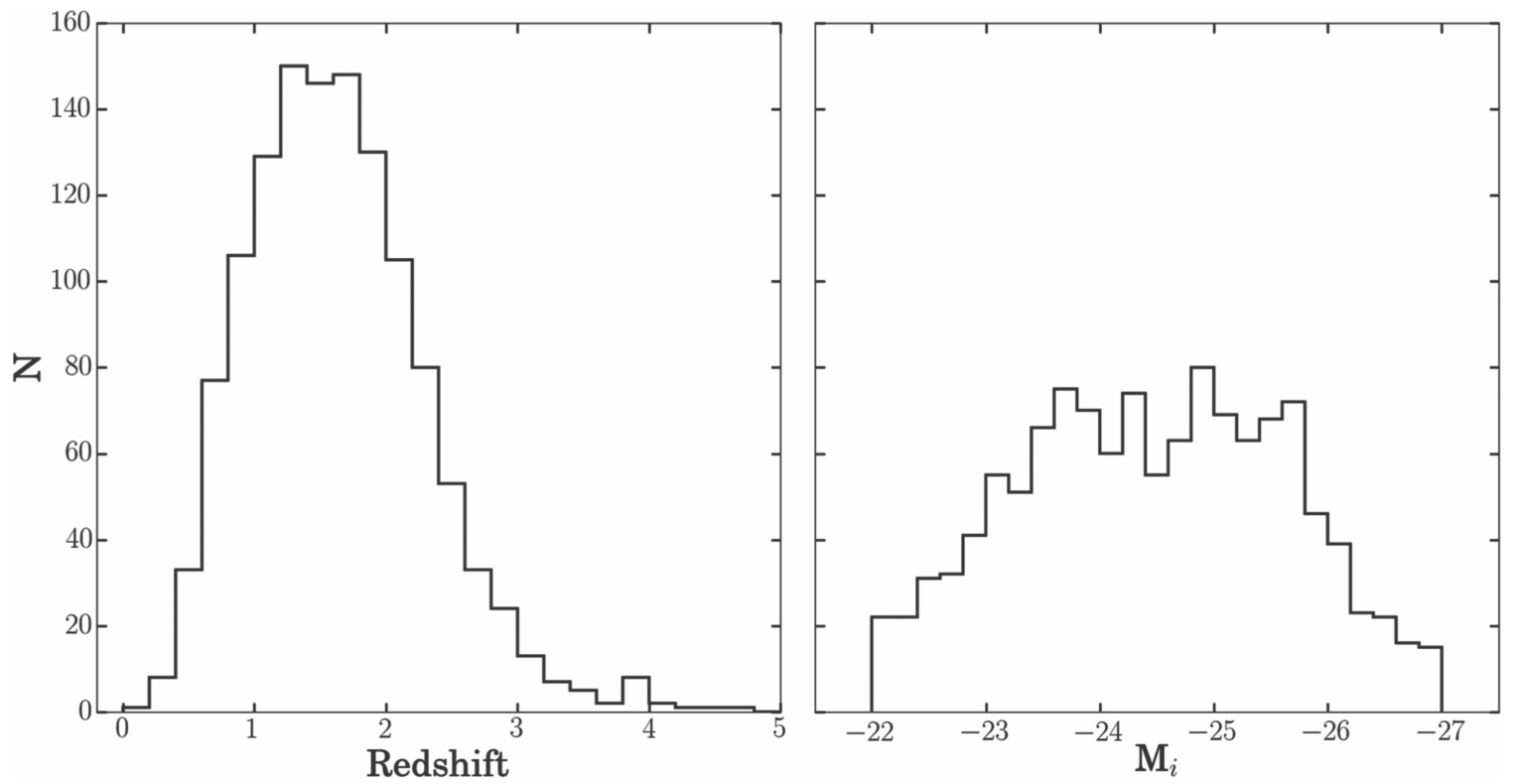

Figure 11. Absolute $i$-band magnitude $M_{i}$ and redshift distributions of the OzDES Quasar Catalog. The quasars are selected to have $M_{i}<-22$ mag and show clear broad/narrow emission/absorption lines in their OzDES spectra.

Table 3

Summary of Quasar Selection Methods Using the P15-S1S2 Quasars

\begin{tabular}{|c|c|c|c|c|c|c|c|c|c|c|c|c|}
\hline \multirow[b]{2}{*}{ Selection } & \multicolumn{2}{|c|}{ Completeness } & \multicolumn{2}{|c|}{ Efficiency } & \multicolumn{2}{|c|}{$C \times E$} & \multicolumn{2}{|c|}{ 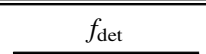 } & \multicolumn{2}{|c|}{$\Sigma_{\mathrm{QSO}}\left(\mathrm{deg}^{-2}\right)$} & \multicolumn{2}{|c|}{$\Sigma_{\Sigma_{\mathrm{All}}\left(\mathrm{deg}^{-2}\right)}$} \\
\hline & Bright & Total & Bright & Total & Bright & Total & Bright & Total & $\begin{array}{c}\text { Bright } \\
\left(\Sigma_{\mathrm{P} 15}=24.4\right)\end{array}$ & $\begin{array}{c}\text { Total } \\
\left(\sum_{\mathrm{P} 15}=132.8\right)\end{array}$ & Bright & Total \\
\hline$\overline{W 1 W 2}$ & 0.91 & 0.93 & 0.71 & 0.54 & 0.65 & 0.50 & 0.96 & 0.55 & 21.3 & 67.9 & 30.0 & 125.8 \\
\hline$g i W 1$ & 0.87 & 0.91 & 0.61 & 0.49 & 0.53 & 0.44 & 0.98 & 0.72 & 20.8 & 87.0 & 34.1 & 177.6 \\
\hline$g i K$ & 0.89 & 0.92 & 0.63 & 0.44 & 0.56 & 0.41 & 0.99 & 0.52 & 21.5 & 63.5 & 34.1 & 144.4 \\
\hline $\mathrm{XDQSOz}$ & 0.92 & 0.90 & 0.73 & 0.60 & 0.67 & 0.54 & 0.96 & 0.55 & 21.5 & 65.7 & 29.5 & 109.6 \\
\hline Variability & 0.86 & 0.88 & 0.088 & 0.11 & 0.075 & 0.10 & 0.86 & 0.90 & 18.0 & 105.2 & 204.7 & 956.1 \\
\hline$W 1 W 2+$ Var & 0.81 & 0.88 & 0.83 & 0.63 & 0.68 & 0.55 & 0.82 & 0.49 & 16.2 & 57.3 & 19.5 & 90.9 \\
\hline$g i W 1+\mathrm{Var}$ & 0.78 & 0.86 & 0.80 & 0.60 & 0.63 & 0.51 & 0.84 & 0.65 & 16.0 & 74.2 & 20.0 & 123.7 \\
\hline $\mathrm{XDQSO}+$ +Var & 0.83 & 0.85 & 0.83 & 0.68 & 0.68 & 0.57 & 0.82 & 0.49 & 16.6 & 55.3 & 20.0 & 81.3 \\
\hline
\end{tabular}

Note. The bright sample refers to sources with $i<19.8$ mag and the total sample refers to sources with $i<22$ mag. Completeness and efficiency are defined at the end of Section 3. $C \times E$ is the product of completeness and efficiency. $f_{\text {det }}$ is the fraction of quasars that meet the selection-dependent detection threshold(s). This fraction is relative to all quasars that satisfy our cuts on point source, flags, and photometric errors. Since both the $W 1 W 2$ and XDQSOz methods have the same DES and WISE detection requirements, their $f_{\text {det }}$ values are the same. The statistical uncertainties in the number of quasars for a typical sample size of 300 (50) in the faint (bright) sample used in this study are of the order of $8 \%(18 \%-22 \%)$. For more discussion, see Section 9 . The last four columns show the surface densities of selected quasars $\left(\Sigma_{\mathrm{QSO}}\right)$ and all candidates $\left(\Sigma_{\mathrm{All}}\right)$ for each method. The surface densities are calculated based on the overall P15 quasar surface density ( $\left.\Sigma_{\mathrm{P} 15}\right), f_{\text {det }}$, and completeness/efficiency as detailed in Section 9 . The surface density of the selected non-quasars is simply $\Sigma_{\text {All }}-\Sigma_{\text {QSO }}$.

and $u-g$ versus $g-r$ in the case of code 256 (a limited amount of $u$-band data was obtained during DES SV in 2012B). We aim to improve the completeness of the OzDES Quasar Catalog as the DES and OzDES observations continue.

\section{Discussion and Summary}

Large samples of quasars are needed to measure quasar luminosity functions and their evolution, particularly at lower luminosities, to estimate the masses of quasar host halos with weak lensing, and to study $\mathrm{BAO}$ with the $\mathrm{Ly} \alpha$ forest, most notably with the Dark Energy Spectroscopic Instrument. ${ }^{55} \mathrm{We}$ used the quasar catalog of Peters et al. (2015) for the SDSS Stripe 82 region that overlaps the DES supernova fields, which we refer to as the P15-S1S2 Quasar Catalog, to analyze the

\footnotetext{
55 http://desi.lbl.gov/tdr/
}

completeness and efficiency of quasar color selection, probabilistic selection, variability selection, and combinations of colors and probabilistic selection methods with variability at bright $(i<19.8 \mathrm{mag})$ and faint $(i<22 \mathrm{mag})$ magnitude limits. We used only point sources that satisfy our data quality and photometric error cuts. The sources used to investigate each method also need to meet the distinct detection thresholds for each selection method because of their dependence on the external WISE and VHS catalogs.

The results of our analyses are summarized in Table 3 and Figure 12. Figure 3 shows the fraction of sources that meet the selection-dependent thresholds (i.e., $f_{\text {det }}$ from Table 3 ) for each selection method as a function of the DES $i$-band magnitude. The completeness and efficiency values both have statistical Poissonian uncertainties due to the actual number of quasars in this study, and systematic uncertainties due to cosmic variance in the quasar population and the characteristics of the DES data 

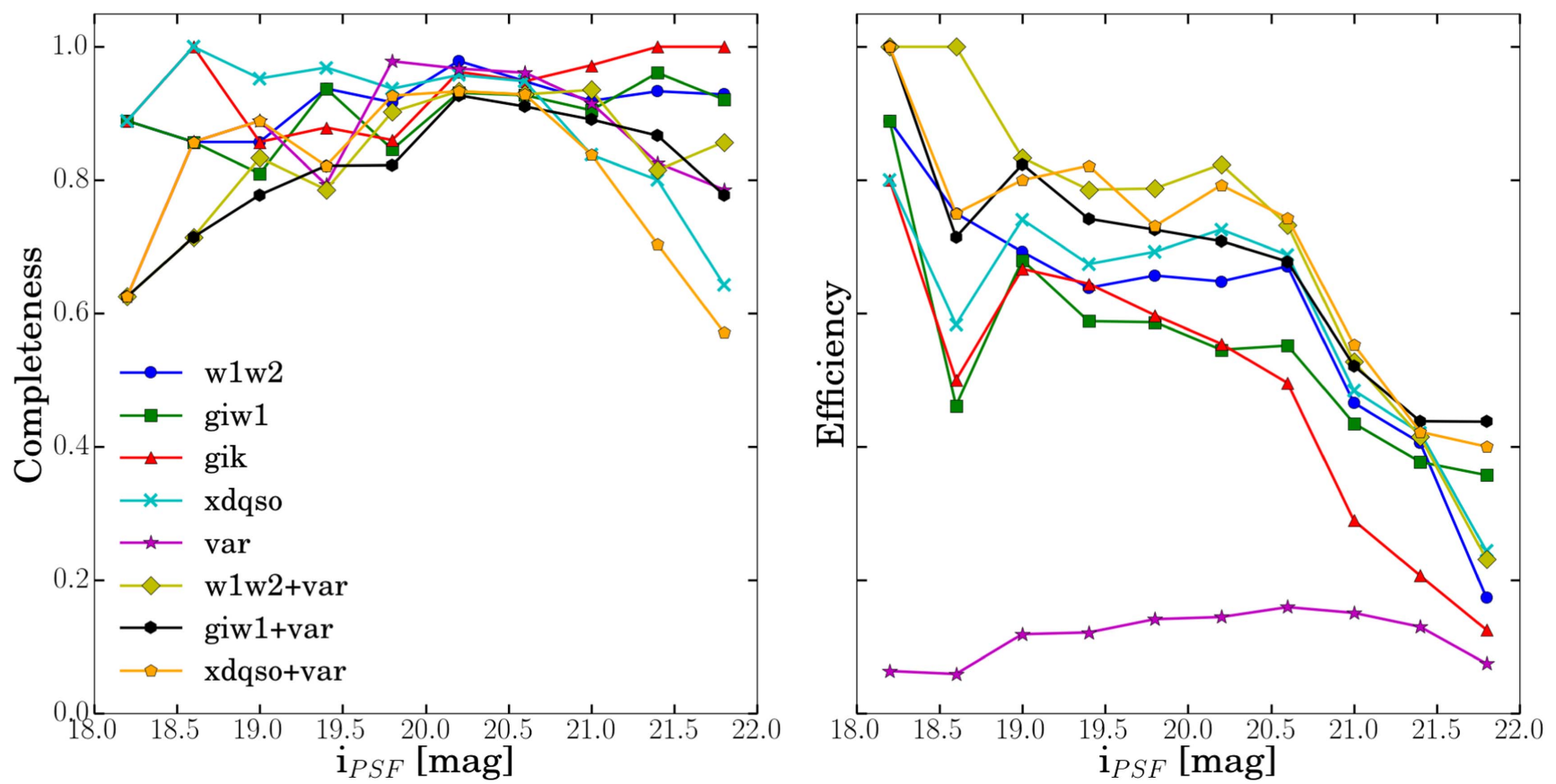

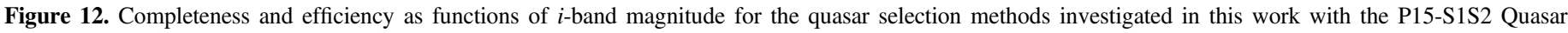
Catalog.

for the S1 and S2 fields. For a typical sample size of 300 (50) quasars in the faint (bright) samples used in this study, the statistical uncertainties are of the order of $8 \%(18 \%-22 \%)$ and these serve as guidelines to compare the relative performance of the selection methods. These percentages correspond to onesided, $90 \%$ confidence limits.

We calculated the surface densities of quasars and all candidates (quasars and non-quasars) selected by each selection method, also shown in Table 3. As the area of Stripe 82 is more precisely known than the overlap between DES S1 and S2 fields with Stripe 82 , and partly because cosmic variance is smaller over a larger region, the surface densities are calculated using the overall P15 quasar surface densities, rather than using the sources in the DES S1 and S2 fields alone. For their "good" quasar candidates (Section 3), the P15 catalog has a surface density $\Sigma_{22}=132.79 \mathrm{deg}^{-2}$ at $i_{\text {SDSS }}<22 \mathrm{mag}$ and $\Sigma_{19.9}=24.36 \mathrm{deg}^{-2}$ at $i_{\text {SDSS }}<19.9 \mathrm{mag}$. The surface density of quasars $\Sigma_{\mathrm{QSO}}$ and all candidates $\Sigma_{\text {All }}$ (quasars and non-quasars) selected by a selection method is then $\Sigma_{\mathrm{QSO}}=\Sigma_{\mathrm{mag}} f_{\mathrm{det}} C$ and $\Sigma_{\mathrm{All}}=\Sigma_{\mathrm{mag}} f_{\mathrm{det}}$ $C / E=\Sigma_{\mathrm{QSO}} / E$, where $\Sigma_{\text {mag }}$ refers to either $\Sigma_{22}$ or $\Sigma_{19.9}, f_{\text {det }}$ is from Table 3, $C$ is the completeness, and $E$ is the efficiency. The surface density of selected non-quasars is simply $\Sigma_{\text {All }}-\Sigma_{\text {Qso }}$.

While variability selection gives the highest surface density, the selection efficiency is one of the lowest and this results in a significant fraction of contaminants. Among individual selection methods, giW1 returns the highest quasar surface density, although it has a lower efficiency than $W 1 W 2$ and $\mathrm{XDQSOz}$. For combined selection methods, $\mathrm{XDQSOz}$ + variability is more efficient than $W 1 W 2+$ variability and $g i W 1+$ variability but also returns lower quasar surface density. The $g i W 1+$ variability selection gives the highest quasar surface density among the three combined selection methods. Taking both the surface densities of selected quasars and selection efficiencies into account, $\mathrm{XDQSOz}$ selection alone and a combination of color or $\mathrm{XDQSO} z$ with variability result in relatively high surface densities of quasars and a modest amount of contamination.

The most significant drawback for both the $W 1 W 2$ and $\mathrm{XDQSOz}$ methods is that the WISE data are only available for $55 \%$ of the quasars with $i<22 \mathrm{mag}$, because both $W 1$ and $W 2$ detections are required. It is possible to apply $\mathrm{XDQSO} z$ without WISE, i.e., using only optical data, where we find the completeness and efficiency to be 51\% (61\%) and $43 \%$ (66\%) for the total $i<22 \mathrm{mag}$ (bright $i<19.8 \mathrm{mag}$ ) sample. This agrees with DiPompeo et al. (2015), who find that the inclusion of WISE data improves the quasar selection. It is also possible to apply XDQSOz with only WISE W1 band by setting the W2 flux to be very small and the errors to be very large. For this, we find a completeness and efficiency of $77 \%(88 \%)$ and $54 \%(73 \%)$. While the giW1 selection method has lower efficiency, it will produce a higher surface density of quasars because a $W 2$ detection is not required.

Since we used only photometry from the first year of DES operations, we employed a simple multi-band $\chi^{2}$ to detect variability. Variability selection based on more sophisticated quasar variability models, such as the damped random walk model, is deferred to the future, when substantially more epochs will be available. The combination of the $W 1 W 2, g i W 1$, or $\mathrm{XDQSO}$ z method with variability improves the efficiency of quasar selection (fewer false positives) at slightly lower completeness. At the bright end, $W 1 W 2+$ variability and $\mathrm{XDQSOz}+$ variability can be applied in the DES supernova fields, but the fraction of sources with WISE detections will diminish for fainter sources. The $g i W 1$ or $g i W 1+$ variability selection method can be used instead, particularly because they both return high quasar surface densities, and in the case of $g i W 1+$ variability, with good selection efficiency. The depth of WISE data is less of an issue for the shallower fields. Although variability selection will not be as useful in these fields because of the small number of epochs, $W 1 W 2$, giW1, or XDQSOz selection would be a good alternative. 
We also presented the OzDES Quasar Catalog of 1263 spectroscopically confirmed quasars in the $30 \mathrm{deg}^{2}$ DES supernova fields brighter than $i=22 \mathrm{mag}$. The catalog includes all the quasars selected from the DES/OzDES reverberation mapping project with good quality OzDES redshifts, $M_{i}<-22 \mathrm{mag}$, and visually confirmed emission and absorption lines. The OzDES Quasar Catalog is not homogeneous or complete, although its completeness will improve as the OzDES observations continue.

Funding for the DES Projects has been provided by the U.S. Department of Energy, the U.S. National Science Foundation, the Ministry of Science and Education of Spain, the Science and Technology Facilities Council of the United Kingdom, the Higher Education Funding Council for England, the National Center for Supercomputing Applications at the University of Illinois at Urbana-Champaign, the Kavli Institute of Cosmological Physics at the University of Chicago, the Center for Cosmology and Astro-Particle Physics at the Ohio State University, the Mitchell Institute for Fundamental Physics and Astronomy at Texas A\&M University, Financiadora de Estudos e Projetos, Fundação Carlos Chagas Filho de Amparo à Pesquisa do Estado do Rio de Janeiro, Conselho Nacional de Desenvolvimento Científico e Tecnológico and the Ministério da Ciência, Tecnologia e Inovação, the Deutsche Forschungsgemeinschaft and the Collaborating Institutions in the Dark Energy Survey.

The Collaborating Institutions are Argonne National Laboratory, the University of California at Santa Cruz, the University of Cambridge, Centro de Investigaciones Energéticas, Medioambientales y Tecnológicas-Madrid, the University of Chicago, University College London, the DES-Brazil Consortium, the University of Edinburgh, the Eidgenössische Technische Hochschule (ETH) Zürich, Fermi National Accelerator Laboratory, the University of Illinois at UrbanaChampaign, the Institut de Ciències de l'Espai (IEEC/CSIC), the Institut de Física d'Altes Energies, Lawrence Berkeley National Laboratory, the Ludwig-Maximilians Universität München and the associated Excellence Cluster Universe, the University of Michigan, the National Optical Astronomy Observatory, the University of Nottingham, The Ohio State University, the University of Pennsylvania, the University of Portsmouth, SLAC National Accelerator Laboratory, Stanford University, the University of Sussex, Texas A\&M University, and the OzDES Membership Consortium.

The DES data management system is supported by the National Science Foundation under Grant Number AST1138766. The DES participants from Spanish institutions are partially supported by MINECO under grants AYA201239559, ESP2013-48274, FPA2013-47986, and Centro de Excelencia Severo Ochoa SEV-2012-0234. Research leading to these results has received funding from the European Research Council under the European Unions Seventh Framework Programme (FP7/2007-2013) including ERC grant agreements 240672, 291329, and 306478.

We would like to thank Richard McMahon and Manda Banerji for helpful suggestions on early drafts of the paper, and the referee for valuable comments.

This work is based in part on data obtained at the Australian Astronomical Observatory through program A/2013B/012. This research made use of Astropy (Astropy Collaboration et al.
2013), a community-developed core Python package for Astronomy (Astropy Collaboration 2013).

\section{References}

Abazajian, K. N., Adelman-McCarthy, J. K., Agüeros, M. A., et al. 2009, ApJS, 182, 543

Aihara, H., Allende Prieto, C., An, D., et al. 2011, ApJS, 193, 29

Arnouts, S., Cristiani, S., Moscardini, L., et al. 1999, MNRAS, 310, 540

Assef, R. J., Kochanek, C. S., Brodwin, M., et al. 2010, ApJ, 713, 970

Assef, R. J., Stern, D., Kochanek, C. S., et al. 2013, ApJ, 772, 26

Astropy Collaboration, Robitaille, T. P., Tollerud, E. J., et al. 2013, A\&A, 558, A33

Banerji, M., Jouvel, S., Lin, H., et al. 2015, MNRAS, 446, 2523

Bernstein, J. P., Kessler, R., Kuhlmann, S., et al. 2012, ApJ, 753, 152

Bertin, E. 2011, in ASP Conf. Ser. 442, Astronomical Data Analysis Software and Systems XX, ed. I. N. Evans et al. (San Francisco, CA: ASP), 435

Bertin, E., \& Arnouts, S. 1996, A\&AS, 117, 393

Blandford, R. D., \& McKee, C. F. 1982, ApJ, 255, 419

Bovy, J., Hennawi, J. F., Hogg, D. W., et al. 2011b, ApJ, 729, 141

Bovy, J., Hogg, D. W., \& Roweis, S. T. 2011a, AnApS, 5, 1657

Bovy, J., Myers, A. D., Hennawi, J. F., et al. 2012, ApJ, 749, 41

Bramich, D. M., Vidrih, S., Wyrzykowski, L., et al. 2008, MNRAS, 386, 887

Butler, N. R., \& Bloom, J. S. 2011, AJ, 141, 93

Chehade, B., Shanks, T., Findlay, J., et al. 2016, MNRAS, 459, 1179

Chung, S. M., Kochanek, C. S., Assef, R., et al. 2014, ApJ, 790, 54

Croom, S. M., Smith, R. J., Boyle, B. J., et al. 2004, MNRAS, 349, 1397

Cushing, M. C., Kirkpatrick, J. D., Gelino, C. R., et al. 2011, ApJ, 743, 50

Dawson, K. S., Schlegel, D. J., Ahn, C. P., et al. 2013, AJ, 145, 10

de Vries, W. H., Becker, R. H., \& White, R. L. 2003, ApJ, 126, 1217

Delubac, T., Bautista, J. E., Busca, N. G., et al. 2015, A\&A, 574, A59

Desai, S., Armstrong, R., Mohr, J. J., et al. 2012, ApJ, 757, 83

Diehl, H. T., Neilsen, E., Gruendl, R., et al. 2016, Proc. SPIE, 9910, 99101D

DiPompeo, M. A., Bovy, J., Myers, A. D., \& Lang, D. 2015, MNRAS, 452, 3124

Drlica-Wagner, A., Bechtol, K., Rykoff, E. S., et al. 2015, ApJ, 813, 109

Erben, T., Hildebrandt, H., Miller, L., et al. 2013, MNRAS, 433, 2545

Flaugher, B. 2005, IJMPA, 20, 3121

Flaugher, B., Diehl, H. T., Honscheid, K., et al. 2015, AJ, 150, 150

Font-Ribera, A., Kirkby, D., Busca, N., et al. 2014, JCAP, 5, 027

Frieman, J. \& Dark Energy Survey Collaboration. 2013, AAS Meeting, 221, 335.01

Heymans, C., Van Waerbeke, L., Miller, L., et al. 2012, MNRAS, 427, 146

Hinton, S. R., Davis, T. M., Lidman, C., Glazebrook, K., \& Lewis, G. F. 2016 , $\mathrm{A} \& \mathrm{C}, 15,61$

Hook, I. M., McMahon, R. G., Boyle, B. J., \& Irwin, M. J. 1994, MNRAS, 268, 305

Ivezić, Ž, \& MacLeod, C. 2014, in IAU Symp. 304, Multiwavelength AGN Surveys and Studies, ed. A. M. Mickaelian \& D. B. Sanders (Cambridge: Cambridge Univ. Press), 395

Ivezic, Z., Tyson, J. A., Abel, B. \& the LSST Collaboration 2008, arXiv:0805.2366

Jannuzi, B. T., \& Dey, A. 1999, in ASP Conf. Ser. 191, Photometric Redshifts and the Detection of High Redshift Galaxies, ed. R. Weymann et al. (San Francisco, CA: ASP), 111

Johnson, H. L., \& Morgan, W. W. 1953, ApJ, 117, 313

Jones, D. H., Saunders, W., Colless, M., et al. 2004, MNRAS, 355, 747

Kaiser, N., Burgett, W., Chambers, K., et al. 2010, Proc. SPIE, 7733, 77330E

Kelly, B. C., Bechtold, J., \& Siemiginowska, A. 2009, ApJ, 698, 895

Kelly, B. C., \& Merloni, A. 2012, AdAst, 2012, 970858

King, A. L., Martini, P., Davis, T. M., et al. 2015, MNRAS, 453, 1701

Kirkpatrick, J. A., Schlegel, D. J., Ross, N. P., et al. 2011a, ApJ, 743, 125

Kirkpatrick, J. D., Cushing, M. C., Gelino, C. R., et al. 2011b, ApJS, 197, 19

Kleinmann, S. G., Lysaght, M. G., Pughe, W. L., et al. 1994, Ap\&SS, 217, 11

Komatsu, E., Smith, K. M., Dunkley, J., et al. 2011, ApJS, 192, 18

Koo, D. C., Kron, R. G., \& Cudworth, K. M. 1986, PASP, 98, 285

Kozłowski, S., Kochanek, C. S., Udalski, A., et al. 2010, ApJ, 708, 927

Lacy, M., Storrie-Lombardi, L. J., Sajina, A., et al. 2004, ApJS, 154, 166

Le Fèvre, Vettolani, G., Garilli, B., et al. 2005, A\&A, 439, 845

Lewis, I. J., Cannon, R. D., Taylor, K., et al. 2002, MNRAS, 333, 279

MacLeod, C. L., Ivezic, Z., Kochanek, C. S., et al. 2010, ApJ, 721, 1014

Maddox, N., Hewett, P. C., Péroux, C., Nestor, D. B., \& Wisotzki, L. 2012, MNRAS, 424, 2876

Mainzer, A., Bauer, J., Cutri, R. M., et al. 2014, ApJ, 792, 30

Mainzer, A., Bauer, J., Grav, T., et al. 2011, ApJ, 731, 53

McMahon, R. G., Banerji, M., Gonzalez, E., et al. 2013, Msngr, 154, 35 
Miller, L., Heymans, C., Kitching, T. D., et al. 2013, MNRAS, 429, 2858

Mohr, J. J., Armstrong, R., Bertin, E., et al. 2012, Proc. SPIE, 8451, 84510D

Mudd, D., Martini, P., Tie, S. S., et al. 2016, MNRAS, submitted (arXiv:1606. 02717)

Myers, A. D., Palanque-Delabrouille, N., Prakash, A., et al. 2015, ApJS, 221, 27 Oke, J. B. 1974, ApJS, 27, 21

Palanque-Delabrouille, N., Yeche, C., Myers, A. D., et al. 2011, A\&A, 530, A122

Peters, C. M., Richards, G. T., Myers, A. D., et al. 2015, ApJ, 811, 95 Peterson, B. M. 1993, PASP, 105, 247

Reed, S. L., McMahon, R. G., Banerji, M., et al. 2015, MNRAS, 454, 3952

Richards, G. T., Fan, X., Newberg, H. J., et al. 2002, AJ, 123, 2945

Richards, G. T., Myers, A. D., Gray, A. G., et al. 2009, ApJS, 180, 67

Richards, G. T., Strauss, M. A., Fan, X., et al. 2006, AJ, 131, 2766

Ross, N. P., Myers, A. D., Sheldon, E. S., et al. 2012, ApJS, 199, 3

Sandage, A., Véron, P., \& Wyndham, J. D. 1965, ApJ, 142, 1307
Schlafly, E. F., \& Finkbeiner, D. P. 2011, ApJ, 737, 103

Schmidt, K. B., Marshall, P. J., Rix, H.-W., et al. 2010, ApJ, 714, 1194

Schneider, D. P., Richards, G. T., Hall, P. B., et al. 2010, AJ, 139, 2360

Sesar, B., Ivezic, Z., Lupton, R. H., et al. 2007, AJ, 134, 2236

Smith, G. A., Saunders, W., Bridges, T., et al. 2004, Proc. SPIE, 5492, 410

Stern, D., Assef, R. J., Benford, D. J., et al. 2012, ApJ, 753, 30

Vanden Berk, D. E., Schneider, D. P., Richards, G. T., et al. 2005, AJ, 129, 2047

Vanden Berk, D. E., Wilhite, B. C., Kron, R. C., et al. 2004, ApJ, 601, 692

Warren, S. J., Hewett, P. C., \& Foltz, C. B. 2000, MNRAS, 312, 827

Wright, E. L., Eisenhardt, P. R. M., Mainzer, A. K., et al. 2010, AJ, 140, 1868

Wu, X.-B., Hao, G., Jia, Z., Zhang, Y., \& Peng, N. 2012, AJ, 144, 49

Xue, Y. Q., Luo, B., Brandt, W. N., et al. 2011, ApJS, 195, 10

Yèche, C., Petitjean, P., Rich, J., et al. 2010, A\&A, 523, A14

York, D. G., Adelman, J., Anderson, J. E., Jr., et al. 2000, AJ, 120, 1579

Yuan, F., Lidman, C., Davis, T. M., et al. 2015, MNRAS, 452, 3047 